\title{
Article \\ Elastic Modeling of Two-Step Transitions in Sterically Frustrated 1D Binuclear Spin-Crossover Chains
}

\author{
Rachid Traiche $^{1}$ (D) Hassane Oubouchou ${ }^{2}$ and Kamel Boukheddaden ${ }^{3, *(D)}$ \\ 1 Département de Physique, Université Hassiba Ben Bouali, BP 78 Ouled Farès, Chlef 02180, Algeria; \\ traiche_rachid@yahoo.fr \\ 2 Laboratoire des Diélectriques, Université Houari Boumedienne, BP 32 El Alia Bab Ezzouar, \\ Alger 16111, Algeria; oh_hassane@yahoo.fr \\ 3 Groupe d'Etudes de la Matière Condensée, Université de Versailles-Saint-Quentin Paris-Saclay, \\ UMR CNRS 8635, 45 Avenue des Etats Unis, 78035 Versailles, France \\ * Correspondence: kamel.boukheddaden@uvsq.fr
}

Citation: Traiche, R.; Oubouchou, H.; Boukheddaden, K. Elastic Modeling of Two-Step Transitions in Sterically Frustrated 1D Binuclear Spin-Crossover Chains. Symmetry 2021, 13, 1836. https://doi.org/ 10.3390/sym13101836

Academic Editors: Sergei Alexandrov and Pierre Yves Manach

Received: 16 June 2021

Accepted: 23 September 2021

Published: 1 October 2021

Publisher's Note: MDPI stays neutral with regard to jurisdictional claims in published maps and institutional affiliations.

Copyright: (c) 2021 by the authors. Licensee MDPI, Basel, Switzerland. This article is an open access article distributed under the terms and conditions of the Creative Commons Attribution (CC BY) license (https:/ / creativecommons.org/licenses/by/ $4.0 /)$.

\begin{abstract}
Among the large family of spin-crossover materials, binuclear systems play an important role due to their specific molecular configurations, allowing the presence of multi-step transitions and elastic frustration. Although this issue benefited from a significant number of spin-based theories, there is almost no elastic description of the spin transition phenomenon in binuclear systems. To overcome this deficiency, in this work we develop the first elastic modeling of thermal properties of binuclear spin-crossover solids. At this end, we investigated a finite spin-crossover open chain constituted of elastically coupled binuclear $(\mathrm{A}=\mathrm{B})$ blocks, $\cdots(\mathrm{A}=\mathrm{B})-(\mathrm{A}=\mathrm{B})-(\mathrm{A}=\mathrm{B}) \cdots$, in which the considered equivalent $\mathrm{A}$ and $\mathrm{B}$ sites may occupy two configurations, namely lowspin (LS) and high-spin (HS) states. The sites of the binuclear unit interact via an intramolecular spring and couple to the neighboring binuclear units via other springs. The model also includes the change of length inside and between the binuclear units subsequent to the spin state changes. When injecting an elastic frustration inside the binuclear unit in the LS state, competing interactions between the intra- and the inter-binuclear couplings emerge. The latter shows that according to the intra- and inter-binuclear elastic constants and the strength of the frustration, multi-step transitions are derived, for which a specific self-organization of type (HS = HS)-(LS-LS)-(HS = HS) $\cdots$ is revealed and discussed. Finally, we have also studied the relaxation of the metastable photoinduced HS states at low temperature, in which two relaxation regimes with transient self-organized states were identified when monitoring the elastic frustration rate or the ratio of intra- and intermolecular elastic interactions. These behaviors are reminiscent of the thermal dependence of the order parameters of the system. The present model opens several possibilities of extensions of elastic frustrations acting in polynuclear spin-crossover systems, which may lead to other types of spin-state self-organizations and relaxation dynamics.
\end{abstract}

Keywords: spin transition; 1D elastic chains; binuclear; bistability and multistability; phase transitions; elastic frustration; Ising models; Monte Carlo simulations

\section{Introduction}

Spin-crossover materials (SCO) emerged recently as one of the new paradigms of switchable molecular solids with serious potential applications in reversible high density memories fast switching molecular electronics as pressure and thermal sensors, in addition to their possible applications in spintronic [1-11]. Among the large families of spin-crossover materials, iron (II)-based SCO solids [1,3,12-17] constitute very interesting objects, due to their bistable vibronic nature which allows them to switch between a nonmagnetic low-spin (LS) state (LS, $\left.e_{g}^{0} t_{2 g}^{6}\right)$ to a paramagnetic high-spin (HS, $\left.e_{g}^{2} t_{2 g}^{4}\right)$ as a function of various stimuli. Among these stimuli are temperature, pressure [18], light [19-23], electric current, static electric field [24-26], high magnetic or pulsed field, etc. [27]. From 
the microscopic point of view, and at the molecular scale, the SCO phenomenon can be considered as originating from a vibronic interaction which couples the electronic and the vibrational structures of the molecule. This results in the electronic switching of the iron center between the LS and the HS states, and is accompanied with a change of the iron-ligands distances. Earlier crystallographic studies $[5,28]$ demonstrated that the volume of the coordination sphere of the molecule expands by almost $30 \%$ during the switching from the HS to LS, while the unit cell volume increases by only 3-5\%. Thus, locally, each conversion of a SCO molecule causes an avalanche of acoustic waves that travel in the crystal and reflect at the surface, which in turn are felt by the other molecules of the lattice. For the thermally-induced SCO transitions, several molecules may switch at the same time in the lattice, each one producing acoustic waves reflecting at the surface and interfering, leading to the generation of complex compressive and tensile stresses, depending on the direction, inside the lattice. This is the physical elastic origin of the long-range interactions between the SCO units, which is clearly directional and may lead to competing interactions. From the experimental point of view, a large variety of thermally-induced spin transition behaviors is observed, among which are the case of (i) continuous spin transitions [29], (ii) discontinuous first-order transitions [30], (iii) incomplete transitions [31] with residual HS fractions and (iv) multi-step spin transitions [32-35]. It is also well known that the spin transition is sensitive to the nature of the ligands [36] as well as to the solvent molecules [37].

Despite these different experimental thermal behaviors, these materials share the same physics. Indeed, it was demonstrated experimentally that changing the ligand or the anion in the material transforms some transitions from first-order to double or three-step transitions [38]. In addition, under applied external pressure, some SCO transitions change from first-order transitions to continuous or incomplete transitions [39]. In particular, the case of two-step transitions attracted a lot of attention, which is clearly the result of the microscopic organization of the HS and LS molecules in the plateau region, and is not due to the existence of some intermediate spin state at the molecular level between the HS and the LS state in Fe(II)-based SCO solids. Interestingly, spin transition materials made of interacting polynuclear SCO, among which the most famous are the dinuclear SCO systems [40,41] usually lead to two-step SCO transitions where the transitions can be continuous, such as for [Fe(2-pic)3]-Cl2.EtOH [42,43], or both are of first-order [44] or even one is gradual and the other is discontinuous [45]. In all these cases, the two transitions are separated by a plateau region, in which the thermal transition strongly "slows down". The thermal width of this plateau usually depends on the elastic rigidity of the chemical bond linking the two iron centers. The presence of the plateau at the transition in the case of dinuclear systems can be explained by steric effects between the two metal centers that hinder the concomitant transition from LS to HS, which involves the increase in the bond-lengths of both transition metals. As a result, the transition of one metal center strongly affects the ligand field of the other, which then delays its transition to a higher temperature. It is also worth noticing that several experimental examples found that the two-step transition can be accompanied by a symmetry breaking [46-52], which results in the emergence of self-organized spin states in the plateau region. In addition, recent reports have shown the existence of multi-steps (three, four and sometimes even more) along the spin transition [49,50,53]. Among these was the famous "Devil's Staircase" [50], in which the number of steps was also enhanced by the presence of inequivalent iron centers. Interestingly, similar behaviors have been also observed in one-dimensional SCO solids [54-56], which means that the elastic frustration from which these phenomena originate presents a common trend.

From the theoretical point of view, spin-crossover materials have been modeled by various type of models. Some of them were first based on Bragg-Williams descriptions of regular solutions leading to the so-called domain model of Sorai and Seki [57], while others were based on the Ising-type description initiated by Wajnflasz and Pick [58,59]. Later, it was demonstrated that these two models are equivalent [60]. 
On the other hand, the modeling of multi-step spin transitions started first with Ising-like models with competing ferro- and antiferro-like interactions $[59,61]$ solved in mean-field approximation or using Monte Carlo method. Very recently, considerable progress has been made towards an elastic description of multi-step transitions phenomena in SCO systems based on the concept of elastic frustration resulting from the presence of antagonist steric effects, which was introduced in ref. [47]. In this paper, the authors have studied the case of a 2D lattice with elastic frustration along the diagonal direction, which shrinks along the LS to HS transition while the unit cell concomitantly expands along the lateral direction.

Later on, in ref. [62], we investigated the case of 1D frustrated elastic SCO mononuclear chains, in which the frustration was introduced through conflicting interactions between the $n n$ and $n n$ equilibrium distances. We have shown in this study that according to the strength of the frustration, several thermodynamic behaviors can be reproduced, among which multi-step [30,49-52,63-69] and incomplete spin transitions are retrieved.

In the present work, we extend the elastic modeling of SCO systems to that of binuclear SCO solids. For this, we first investigate the thermodynamic properties of an isolated open 1D chain made of elastically coupled SCO binuclear units $(A=B)$, where both A and B can switch from the LS to HS with temperature. The sites A and B are coupled via an intramolecular spring, and coupled to the other sites belonging to neighboring binuclear units through other springs. To broaden the horizons of the model, an elastic frustration is injected in the intramolecular part, which results in competing interactions between the intra- and the inter-binuclear couplings.

This electro-elastic Hamiltonian, which includes both spins and positions variables, is solved using Monte Carlo (MC) simulations preformed on both degrees of freedom. Analytical calculations based on a homogeneous elastic description of the chain are also developed to validate the MC results. The results show that the misfit of intra- and interbinuclear elastic interactions is already sufficient to create an elastic frustration between the binuclear units, leading to stabilization of intermediate states when moving from the LS to the HS state. These intermediate states display the emergence of spatial self-organization of the spin states, in which the binuclear units are coupled antiferro while the organization inside the binuclear is of ferro type. Similar results are obtained by monitoring the strength of the elastic frustration.

The manuscript is organized as follows. Section 2 introduces the model Hamiltonian and the details of the simulations; in Section 3, we present the results related to the equilibrium properties and derive the phase diagrams; in Section 4, we present the behavior of the isothermal relaxation of the metastable HS for various intra and intermolecular elastic constants and frustration rates; and in Section 5 we conclude and outline some possible extensions of the model.

\section{The Model Hamiltonian}

The present SCO model for binuclear lattices is inspired from our previous elastic modeling [70-72] of the spin-crossover material. Let us first recall the basic concepts of the elastic modeling of SCO materials, accounting for their spin state and volume degrees of freedom. In the case of 1D SCO systems, the Hamiltonian contains electronic (spin state) and lattice (elastic) contributions.

In this work, we consider a spin-crossover chain (Figure 1) constituted of elastically coupled binuclear units, $\mathrm{A}=\mathrm{B}$, in which $\mathrm{A}$ and $\mathrm{B}$ sites may occupy two configurations, namely, low-spin (LS) and high-spin (HS). Using the same ideas of Ising model, each spin state of the molecule is associated with a fictitious two-state spin operator, $S_{i}$. Thus, $S_{i}=+1$ will correspond to the HS state, while the value $S_{i}=-1$ will be associated with the LS state. The sites are connected by springs and are supposed to move only along the chain, i.e., in the $x$ direction. Thus, the binuclear at site $i$ contains two atoms $\mathrm{A}$ and $\mathrm{B}$ with positions $x_{i}^{\mathrm{A}}$ and $x_{i}^{\mathrm{B}}$, and their associated spin states are denoted with $S_{i}^{\mathrm{A}}$ and $S_{i}^{\mathrm{B}}$. 


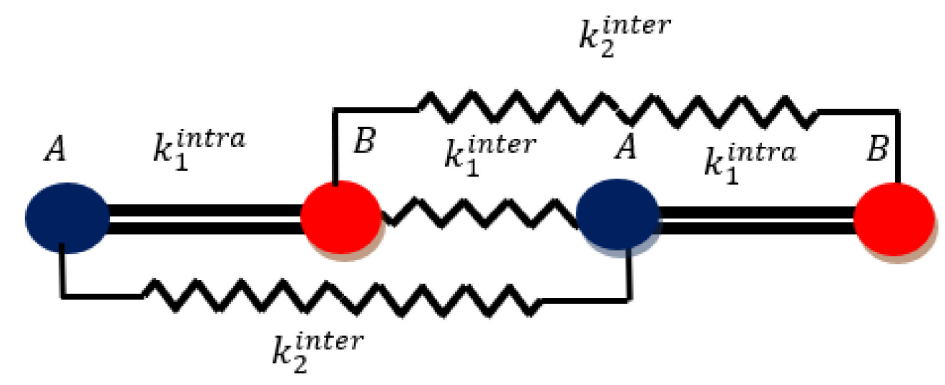

Figure 1. Schematic view of two coupled binuclear units showing the intra- and inter-binuclear elastic interactions. $k_{1}^{\text {intra }}$ is the intramolecular elastic constant, while $k_{1}^{\text {inter }}$ and $k_{2}^{\text {inter }}$ are the $n n$ intermolecular and nnn elastic constants. The bond lengths distances between the sites depend on the spin states of the connected sites.

Each site is connected elastically to its nearest neighbors (nn) by a spring of elastic constant $k_{1}^{\text {intra }}$ inside the binuclear unit, by $k_{1}^{\text {inter }}$ between the binuclear units and to its next nearest neighbors by another spring of constant elastic $k_{2}^{i n t e r}$, as schematized in Figure 1.

The equilibrium bond length connecting two $n n$ sites $i$ and $j$ is noted as $R_{e q}^{n n}\left(S_{i}, S_{j}\right)$. This quantity can be written as a function of the spin states, so as to have a bigger length of the chain in the HS state in comparison with that of the LS state. In the same way, the equilibrium distance between two $n n n$ sites $i$ and $k$, denoted $R_{e q}^{n n n}\left(S_{i}, S_{k}\right)$, is also a function of the spin states, $S_{i}$ and $S_{k}$.

The Hamiltonian describing this 1D systems, taking into account the electronic and elastic degrees of freedom of the lattice, is derived from our previous 2D electro-elastic model [71], which has been investigated for several situations of crystal shapes and sizes [72-79], including core-shell [80] and elastically-frustrated lattices [47,62]. The Hamiltonian of this 1D version designed for 1D binuclear chain of Figure 1 writes,

$$
\begin{gathered}
H=\sum_{i} \frac{1}{2}\left[\Delta_{0}-k_{\mathrm{B}} T \ln (g)\right]\left(S_{i}^{\mathrm{A}}+S_{i}^{\mathrm{B}}\right)+k_{1}^{i n t r a} \sum_{i=1}\left[\left(x_{i}^{\mathrm{B}}-x_{i}^{\mathrm{A}}\right)-R_{0}^{n n}\left(S_{i}^{\mathrm{A}}, S_{i}^{\mathrm{B}}\right)\right]^{2} \\
+k_{1}^{i n t e r} \sum_{i=1}\left[\left(x_{i+1}^{\mathrm{A}}-x_{i}^{\mathrm{B}}\right)-R_{0}^{n n}\left(S_{i+1}^{\mathrm{A}}, S_{i}^{\mathrm{B}}\right)\right]^{2}+k_{2}^{i n t e r} \sum_{i}\left[\left(x_{i+1}^{\mathrm{A}}-x_{i}^{\mathrm{A}}\right)-R_{0}^{n n n}\left(S_{i+1}^{\mathrm{A}}, S_{i}^{\mathrm{A}}\right)\right]^{2} \\
+k_{2}^{\text {inter }} \sum_{i}\left[\left(x_{i+1}^{\mathrm{B}}-x_{i}^{\mathrm{B}}\right)-R_{0}^{n n n}\left(S_{i}^{\mathrm{B}}, S_{i+1}^{\mathrm{B}}\right)\right]^{2}
\end{gathered}
$$

The first term of Hamiltonian (1) represents the effective ligand-field energy, which here is temperature-dependent due to the presence of the degeneracy ratio, $g$, between the HS and the LS states. Thus, the total contribution, writes, $\Delta_{0}-k_{\mathrm{B}} T \ln (g)$, where $\Delta_{0}$, is 0 $\mathrm{K}$ ligand field energy and $k_{\mathrm{B}} \ln (g)$, is the entropy of the HS states, which originates from electronic and vibrational degrees of freedom. The molar entropy change at the transition, $\Delta S=R \ln g$, easily derived from calorimetric data [34], allows the determination of $g$ values, which are found as ranging from 100 to 2000. The second term of the Hamiltonian refers to the intramolecular elastic interactions inside the binuclear units, while the third term accounts for the elastic inter-binuclear A-B closest atoms (see Figure 1), which can be considered here as nearest neighbors (nn). The fourth and the fifth terms of Equation (1) describe the elastic interactions between next nearest neighbors ( $n n n)$ atoms of type (A-A and B-B) belonging to different binuclear sites. Here, all elastic constants, $k_{1}^{\text {intra }}, k_{1}^{\text {inter }}$ and $k_{2}^{\text {inter }}$ are assumed, for simplicity, as independent on spins and lattice positions. Furthermore, for simplicity, the ligand fields, $\Delta_{0}$, and degeneracies, $g$, of $A$ and $B$ sites are assumed to be equal.

\subsection{The Equilibrium Distances}

The molecules (here, the sites) interact via elastic springs, as depicted in Figure 1, and the equilibrium bond lengths between two $n n i$ and $i \pm \mathbf{1}$ (resp. nnn. $i$ and $i \pm 2$ ) sites with spins $S_{i}^{\mathrm{A}}$ and $S_{i}^{\mathrm{B}}$ (resp. $S_{i}^{\mathrm{A}}$ and $S_{j}^{\mathrm{A}}, S_{i}^{\mathrm{B}}$ and $S_{j}^{\mathrm{B}}$ ) are noted $R_{0}^{n n}\left(S_{i}^{\mathrm{A}}, S_{i}^{\mathrm{B}}\right)$ (resp. $R_{0}^{n n n}\left(S_{i}^{\mathrm{A}}, S_{j}^{\mathrm{A}}\right)$ and $\left.R_{0}^{n n n}\left(S_{i}^{\mathrm{B}}, S_{j}^{\mathrm{B}}\right)\right)$. We denote by $R_{0}^{H H}, R_{0}^{L L}$ and $R_{0}^{H L}$, the equilibrium distances between $n n$ HS-HS, LS-LS and HS-LS sites. Then, we have $R_{0}^{H H}=R_{0}(+1,+1)$, 
$R_{0}^{L L}=R_{0}(-1,-1)$ and $R_{0}^{H L}=R_{0}(+1,-1)$. Moreover, we assume the following relation, $R_{0}^{H L}=\frac{1}{2}\left(R_{0}^{H H}+R_{0}^{L L}\right)$, which states that the equilibrium bond length between HS-LS configuration is equal to the average of those of LS-LS and HS-HS configurations. Using the previous parameters, $R_{0}^{H H}, R_{0}^{L L}$ and $R_{0}^{H L}$, it is straightforward to demonstrate that general relations connecting the equilibrium bond lengths and the spin states are written as,

$$
\begin{gathered}
R_{0}^{n n}\left(S_{i}^{\mathrm{A}}, S_{i}^{\mathrm{B}}\right)=R_{0}^{H L}+\frac{\delta_{R}}{2}\left(S_{i}^{\mathrm{A}}+S_{i}^{\mathrm{B}}\right) \\
R_{0}^{n n n}\left(S_{i}^{\mathrm{A}}, S_{j}^{\mathrm{A}}\right)=2 R_{0}^{H L}+\delta_{R}\left(S_{i}^{\mathrm{A}}+S_{j}^{\mathrm{A}}\right)=2 R_{e q}^{n n}\left(S_{i}^{\mathrm{A}}, S_{i}^{\mathrm{A}}\right) \\
R_{0}^{n n n}\left(S_{i}^{\mathrm{B}}, S_{j}^{\mathrm{B}}\right)=2 R_{0}^{H L}+\delta_{R}\left(S_{i}^{\mathrm{B}}+S_{j}^{\mathrm{B}}\right)=2 R_{e q}^{n n}\left(S_{i}^{\mathrm{B}}, S_{i}^{\mathrm{B}}\right)
\end{gathered}
$$

where $\delta_{R}=\left(R_{0}^{H H}-R_{0}^{L L}\right)$ represents the lattice parameter misfit between the LS and HS states.

\subsection{Elastic Frustration inside the Binuclear Units}

The concept of elastic frustration supposes the existence of a lattice mismatch between the $n n$ and $n n n$ bond lengths, leading to antagonist equilibrium distances. From the experimental point of view, these behaviors may result from the existence of steric effects imposed by the interactions between ligands of neighboring molecules, due to their extended spatial occupations [34,60,81]. For example, a strong $\pi-\pi$ stacking interaction may contribute to cooperative ferro-elastic interactions, while other electrostatic interactions such as "weak" hydrogen bonding may prevent the molecular volume change along the transition between LS and HS, leading to antiferro-elastic interactions.

For this purpose, we chose the HS state free from any elastic frustration, while we inject an elastic frustration between the $n n$ and $n n n$ for the other HS $=$ LS and LS $=$ LS configurations. In particular, in the present study, the elastic frustration is considered only inside the binuclear units as follows,

$$
R_{e q}^{n n}\left(S_{i}^{\mathrm{A}}, S_{i}^{\mathrm{B}}\right)=R_{0}^{n n}\left(S_{i}^{\mathrm{A}}, S_{i}^{\mathrm{B}}\right)+\frac{\delta_{R} \xi}{2}\left(1-\frac{\left(S_{i}^{\mathrm{A}}+S_{i}^{\mathrm{B}}\right)}{2}\right)
$$

where $\xi>0$ is the frustration strength.

Using expression (5), the HS state $\left(S_{i}^{\mathrm{A}}=+1, S_{i}^{\mathrm{B}}=+1 \forall i\right)$ is indeed non-frustrated, since $R_{e q}^{n n}(+1,+1)=R_{0}^{H H} \forall \xi$. When $\xi>0$, the equilibrium distances between $n n$ pairs for $\mathrm{L}=\mathrm{L}$ and $\mathrm{H}=\mathrm{L}$ configurations are respectively,

$$
R_{e q}^{n n}(-1,-1)=R_{0}^{L L}+\delta_{R} \xi \text { and } R_{e q}^{n n}( \pm 1, \mp 1)=R_{0}^{H L}+\frac{\delta_{R}}{2} \xi
$$

while those of intermolecular $n n n$ sites are maintained to $2 R_{0}^{L L}$ and $2 R_{0}^{H L}$, respectively.

Table 1 summarizes the sixth spin configurations of a pair of neighboring binuclear molecules one and two. It is clear from Table 1 that when the system is in the LS phase, the elastic frustration strength $\xi$ leads to antagonist distances between $n n$ and $n n n$ distances. Indeed, since the sites are restricted to move only along the direction of the chain, the nnn distance for example between A1 and A2 should be equal to the sum of the distances between A1-B1 and B1-A2. This is not the case when the frustration is injected as we can see for the LS case, where the total nnn equilibrium distance $\left(\mathrm{LS}_{1}-\mathrm{LS}_{2}\right.$, see Table 1$)$ is equal to $2 R_{0}^{L L}$ (no frustration), while the $n n$ distances impose a total value equal to $2 R_{0}^{L L}+\delta_{R} \xi$. As a result, the system will try to share the elastic energy excess with the other bonds in order to optimize the spatial distribution of elastic energy, avoiding large compressive and tensile stresses. Obviously, the resulting distribution of bond length distance will depend on the strengths of the $n n$ and $n n n$ elastic constants. 
Table 1. Nearest neighbors and next nearest neighbors spin configurations of a pair of binuclear units (1 and 2$)$ and their corresponding equilibrium distances showing their antagonist character, except for $\xi=0$, where $n n$ and $n n n$ distances fit each other.

\begin{tabular}{ccccc}
\hline Spin Configurations & \multicolumn{1}{c}{ nn Equilibrium Distances } & nnn Equilibrium Distances \\
\hline $\mathrm{HS}_{1}=\mathrm{HS}_{1}-\mathrm{HS}_{2}=\mathrm{HS}_{2}$ & $R_{0}^{H H}$ & $R_{0}^{H H}$ & $R_{0}^{H H}$ & $2 R_{0}^{H H}$ \\
$\mathrm{LS}_{1}=\mathrm{LS}_{1}-\mathrm{LS}_{2}=\mathrm{LS}_{2}$ & $R_{0}^{L L}+\delta_{R} \xi$ & $R_{0}^{L L}$ & $R_{0}^{L L}+\delta_{R} \xi$ & $2 R_{0}^{H H}$ \\
$\mathrm{HS}_{1}=\mathrm{HS}_{1}-\mathrm{HS}_{2}=\mathrm{LS}_{2}$ & $R_{0}^{H H}$ & $R_{0}^{H H}$ & $R_{0}^{H L}+\frac{\delta_{R}}{2} \xi$ & $2 R_{0}^{H H}$ \\
$\mathrm{HS}_{1}=\mathrm{HS}_{1}-\mathrm{LS}_{2}=\mathrm{LS}_{2}$ & $R_{0}^{H H}$ & $R_{0}^{H L}$ & $R_{0}^{L L}+\delta_{R} \xi$ & $2 R_{0}^{H L}$ \\
$\mathrm{HS}_{1}=\mathrm{LS}_{1}-\mathrm{LS}_{2}=\mathrm{LS}_{2}$ & $R_{0}^{H L}+\frac{\delta_{R}}{2} \xi$ & $R_{0}^{L L}$ & $R_{0}^{L L}+\delta_{R} \xi$ & $2 R_{0}^{H L}$ \\
$\mathrm{HS}_{1}=\mathrm{LS}_{1}-\mathrm{HS} S_{2}=\mathrm{LS}_{2}$ & $R_{0}^{H L}+\frac{\delta_{R}}{2} \xi$ & $R_{0}^{H L}$ & $R_{0}^{H L}+\frac{\delta_{R}}{2} \xi$ & $2 R_{0}^{H L}$ \\
\hline
\end{tabular}

We start by studying the frustration for a "homogeneous" binuclear chain, i.e., $k_{1}^{\text {intra }}=k_{1}^{\text {inter }}$. In the simulations, we considered the following values for the nonfrustrated equilibrium distances, $R_{0}^{L L}=1 \mathrm{~nm}, R_{0}^{H H}=1.2 \mathrm{~nm}, R_{0}^{H L}=1.1 \mathrm{~nm}$, leading to the lattice misfit value, $\delta_{R}=0.2 \mathrm{~nm}$. The $n n$ elastic constants have been fixed to $k_{1}^{\text {intra }}=k_{1}^{\text {inter }}=34,000 \mathrm{~K} \cdot \mathrm{nm}^{-2}=34 \mathrm{meV} \cdot \mathrm{A}^{-2}$, and the $n n n$ elastic constant is taken as $k_{2}^{\text {inter }}=0.1 k_{1}^{\text {inter }}$ while $\Delta_{0}=450 \mathrm{~K}$ and $\ln g=5$. This value leads to the entropy change, $\Delta S=41 \mathrm{~J} \cdot \mathrm{K} \cdot \mathrm{mol}^{-1}$, a value which is in fair agreement with experimental finding of calorimetry [32].

\section{Results and Discussion}

In the electro-elastic model for SCO materials, the spin transition is described using two macroscopic order parameters, which are the HS fraction, $n_{H S}$, connecting to the average magnetization $\langle S\rangle$, and the average lattice bond length, $\langle d\rangle$. Another quantity of interest, $\delta n=\frac{1}{2}\left(<S_{i+1}^{\mathrm{A}}-S_{i}^{\mathrm{A}}>\right)\left(\right.$ or $\left.\frac{1}{2}\left(<S_{i+1}^{\mathrm{B}}-S_{i}^{\mathrm{B}}>\right)\right)$, which measures the degree of order between $A(B)$ sites belonging to consecutive binuclear units, is also used. The intramolecular information about the binuclear units can be also studied by monitoring the temperature dependence of the quantities $\left\langle d_{\text {intra }}>\right.$ and $\left\langle d_{\text {inter }}\right\rangle$, the average of intra and inter binuclear distances. These quantities can be expressed simply as,

$$
\begin{gathered}
n_{H S}=\frac{1+<S>}{2}, \delta n=\frac{\frac{1}{2} \sum_{i=2}^{N / 2}\left(S_{i+1}^{\mathrm{A}}-S_{i}^{\mathrm{A}}\right)}{\left(\frac{N-1}{2}\right)},<d_{\text {intra }}>=\frac{\sum_{i=1}^{\frac{N}{2}-1}\left|x_{i}^{\mathrm{B}}-x_{i}^{\mathrm{A}}\right|}{\left(\frac{N-1}{2}\right)}, \\
<d_{\text {inter }}>=\frac{\sum_{i=2}^{N / 2}\left(\left|x_{i+1}^{\mathrm{A}}-x_{i}^{\mathrm{B}}\right|\right.}{\left(\frac{N-1}{2}\right)}, \text { and }\left\langle d>=\frac{<d_{\text {intra }}>+<d_{\text {inter }}>}{2}\right.
\end{gathered}
$$

where $\langle S\rangle$ is the average spin value and $\left|x_{i}^{\mathrm{B}}-x_{i}^{\mathrm{A}}\right|$ is the distance between neighboring sites $\mathrm{A}$ and $\mathrm{B}$ inside the same binuclear unit at position $i$. This is while $\left|x_{i+1}^{\mathrm{A}}-x_{i}^{\mathrm{B}}\right|$ is the distance between neighboring $\mathrm{B}$ (in binuclear $i$ ) and A (inside binuclear $i+1$ ) sites belonging to consecutive binuclear units.

The Hamiltonian (1), which includes spin and distortion variables, cannot be solved analytically in view of its complex structure. Therefore, we use the Monte Carlo (MC) technique to study its thermal properties with frustration, the strength of which is controlled by the parameter, $\xi$.

The investigations of the physical properties of the adapted electro-elastic 1D model for binuclear solids is implemented on a chain with $N=60$ atoms, consisting in 30 binuclear units. Here, each SCO atom interacts with its surrounding nearest and next nearest neighbors. When starting the simulations, we first prepare the system in the HS (nonfrustrated by construction) phase by fixing all the spins to $S_{i}=+1$ and all $n n$ lattice distances equal to $R_{0}^{H H}$. The Monte Carlo simulations, based on the Metropolis algorithm, concerned both spin and lattice position variables. The stochastic algorithm is performed in the following way: first we select randomly a site, $i$, with spin $S_{i}$ and position $x_{i}$, and we change its spin state to $S_{i}^{\prime}=-S_{i}$ without position change. This new state is accepted or rejected by the usual Metropolis criterion. In the second step, whatever the result of the first 
MC process, we mechanically relax the lattice by a slight motion of all the nodes randomly selected, with a quantity $\delta x=0.001 \mathrm{~nm}$, which is rather small compared to the distance between two consecutive sites (average distance $\sim 1.1 \mathrm{~nm}$ ). This procedure is repeated 10 times for each spin flip. When all nodes of the lattice are visited for the spin change, we define the unit Monte Carlo step, denoted "MCS". The same procedure is performed at each temperature. To determine the thermal properties of the system, we first cool down from 140 to $1 \mathrm{~K}$ and then warm up to $140 \mathrm{~K}$, with a $1 \mathrm{~K}$ increment. At each temperature, we perform $10^{5}$ MCS to reach the equilibrium state and we use $10^{5}$ other MCS for the statistics. Using the parameter values of the ligand field energy, $\Delta_{0}$, and the degeneracy ratio, $g$, and according to the general law that at the transition the total effective ligand field becomes equal to zero, we arrive immediately to the expression of the transition temperature $T_{e q}^{(0)}$, through the relations: $\langle S\rangle=0\left(n_{H S}=0.5\right)$ and $\langle d\rangle=R_{0}^{H L}$,

$$
T_{e q}^{(0)}=\frac{\Delta_{0}}{k_{\mathrm{B}} \ln (g)}
$$

Figure 2 summarizes the thermal behavior of the HS fraction values for the elastic constant's values, $k_{1}^{\text {intra }}=k_{1}^{\text {inter }}=34,000 \mathrm{~K} \cdot \mathrm{nm}^{-2}=34 \mathrm{meV} \cdot \mathrm{A}^{-2}$, for the frustration rate, $\xi=0$. We see clearly that the chain presents a first-order transition between the LS and HS states with a large thermal hysteresis, which is characteristic of a strong cooperative SCO system. The MC equilibrium temperature is evaluated by taking $T_{e q}^{M C}=\frac{T^{+}+T^{-}}{2} \approx 90 \mathrm{~K}$, where $T^{+}$and $T^{-}$are the upper and lower transition temperatures at $n_{H S}=0.5$.
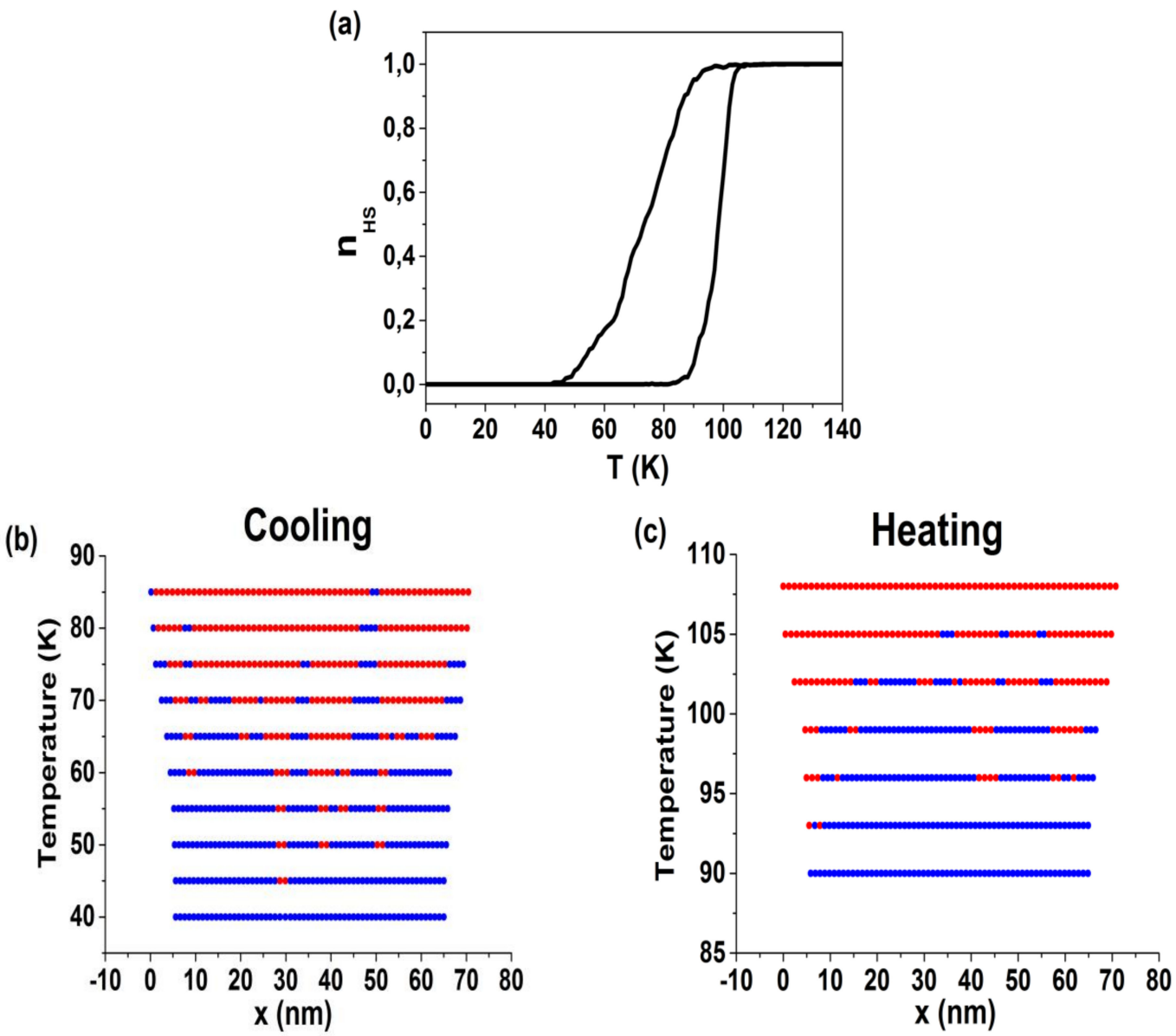

Figure 2. (a) Thermal dependence of the HS fraction, $n_{H S}$, in the non-frustrated case $(\xi=0)$ for strong couplings $k_{1}^{\text {intra }}=k_{1}^{\text {inter }}=34,000 \mathrm{~K} \cdot \mathrm{nm}^{-2}$ leading to a hysteretic behavior. $(\mathbf{b}, \mathbf{c})$ are corresponding snapshots of the spin state configurations of the lattice along the thermal transition of heating and cooling, respectively. The other parameter values are: $R_{0}^{L L}=1 \mathrm{~nm}, R_{0}^{H H}=1.2 \mathrm{~nm}, R_{0}^{H L}=1.1 \mathrm{~nm}, \delta_{R}=0.2 \mathrm{~nm}, \Delta_{0}=450 \mathrm{~K}$ and $\ln g=5$. 
The results of Figure 2 indicate the presence of a thermal hysteresis curve while we deal with a 1D system. This effect can be attributed to the long-range character of the elastic interactions, which belong to mean field universality class, as demonstrated by Miyashita et al. [81] and as confirmed in many recent works [82] related to the study of the dynamic phase transition in 1D. These investigations demonstrated that the hysteresis widths increase with the length of the chain.

The strength of the cooperative interactions is proportional to the misfit of elastic energy per site, $\left(k_{1}^{i n t r a}+2 k_{2}^{\text {inter }}\right) \delta_{R}^{2}$. It is worth noticing here that this behavior contrasts with that of the usual Ising $1 \mathrm{D}$ chains with short-range interactions, the kinetic hysteresis of which are significantly less wide. One may think that the presence of elastic interactions, which are known to lead to long-range interactions, clearly lead to larger energy barriers of metastable phases, which may then enhance the existence of these larger kinetic thermal hysteresis. In experimental systems of 1D spin-crossover systems [66], the SCO chains are rarely isolated and several types of interactions, such as weak hydrogen bonding or strong stacking $\pi-\pi$, may connect them, which then lead to 3D topology of interactions with a strong 1D character, due to this structural anisotropy. In such situations, true first-order transitions or very sharp transitions are allowed.

The spatial distribution of the HS and LS species along the cooling and heating processes of the phase transition are summarized in Figure 2b,c. Here, we see that the transformation starts from both extremities of the chain without showing the presence of any special self-organization.

Figure 3a presents the thermal evolution of the HS fraction for different values of the frustration rate $\xi$. We noticed that in the absence of frustration, the SCO transition is of first-order with hysteresis (black curves of Figure 3a), with a width of $\Delta T=21 \mathrm{~K}$. In contrast, when the elastic frustration between $n n$ and $n n n$ sites comes into play, the HS fraction curve shifts to a lower temperature region, and the previous first-order transition transforms to a non-hysteretic two-step transition with a large plateau around $n_{H S}=0.5$ (pink curves of Figure 3a). A further increase of the frustration strength enlarges the plateau and stabilizes the intermediate state. Similar information is also obtained from the thermal dependence of the average lattice parameter, $\langle d\rangle$, given in Figure $3 b$, which exhibits a sizable and continuous increase in $\langle d\rangle_{L L}$ in the LS state. In particular, it is remarked that the plateau does emerge in the simulations from the threshold value $\xi=0.6$, for which an average distance corresponds, $\left\langle d>_{L L} \simeq 1.09 \mathrm{~nm}\left(\sim R_{0}^{H L}\right)\right.$. The analysis of the thermal behavior of the average intra- and inter-dinuclear distances, $\left\langle d_{\text {intra }}\right\rangle$ and $\left\langle d_{\text {inter }}\right\rangle$, given in Figure $3 c$,d, leads to the same behavior as that of Figure $3 b$, due to the homogenous character of all equilibrium HS-HS, HS-LS and LS-LS distances considered inside the binuclear and between the binuclear species.

In Figure 3e, we plotted the corresponding thermal dependence of the order parameter $\delta n$, given in Equation (7), which quantifies the degree of ordering between consecutive binuclear units. In the HS and LS states, one has $\delta n=0$, which means that sublattices $\mathrm{A}$ and $\mathrm{B}$ are equivalent. In contrast, in the plateau region where $\left\langle S_{\mathrm{A}}+S_{\mathrm{B}}\right\rangle=0$ (i.e., $\left.n_{H S}=0\right)$, the curves $\delta n(T)$ reach a maximum indicating the existence of LS $=$ LS-HS $=$ HS ordering. This order is partial for $\xi=0.2$ and becomes almost complete in the whole plateau region for $\xi=1$, indicating the existence of a symmetry breaking between the sublattices which couple in an antiferro-elastic fashion.

Figure $3 \mathrm{f}$ summarizes the spatial configurations of the spin states (red dots $=\mathrm{HS}$ and blue dots = LS) inside the lattice along the thermal transition of the two-step behavior obtained for $\xi=1$. Here, in the temperature interval $34 \leq T \leq 45 \mathrm{~K}$, we see the emergence of self-organization of spin states inside the plateau region under the form of spin configurations LS = LS-HS = HS-LS = LS-HS = HS, noted for simplicity L2H2 . . , with the presence of antiphase boundaries. The latter take place because the symmetric configuration H2L2 has the same energy. It is interesting to remark that while these configurations occur in spin crossover molecules coupled ferro elastically inside the binuclear units, the binuclear units themselves are coupled in an antiferro fashion. 
(a)

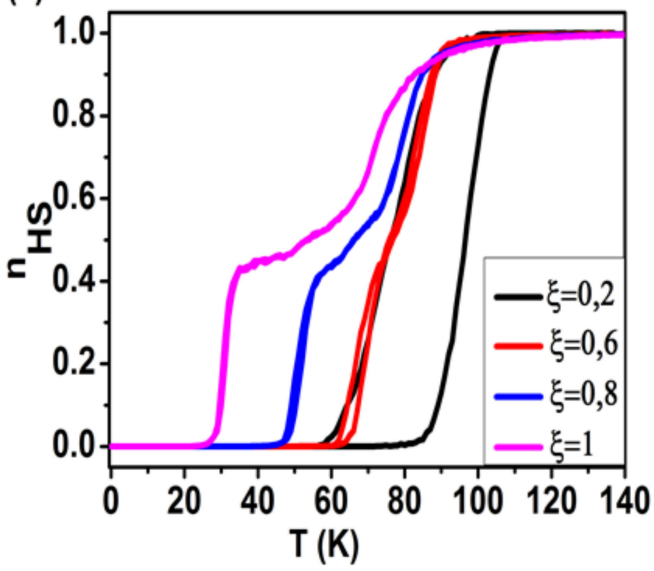

(c)

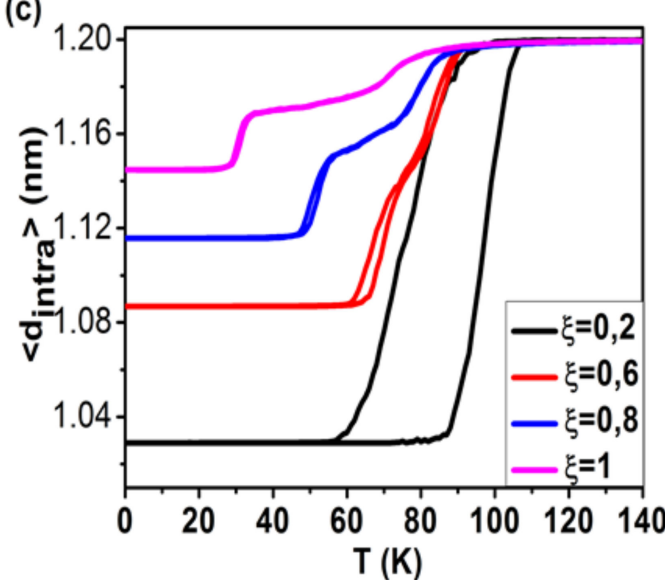

e)

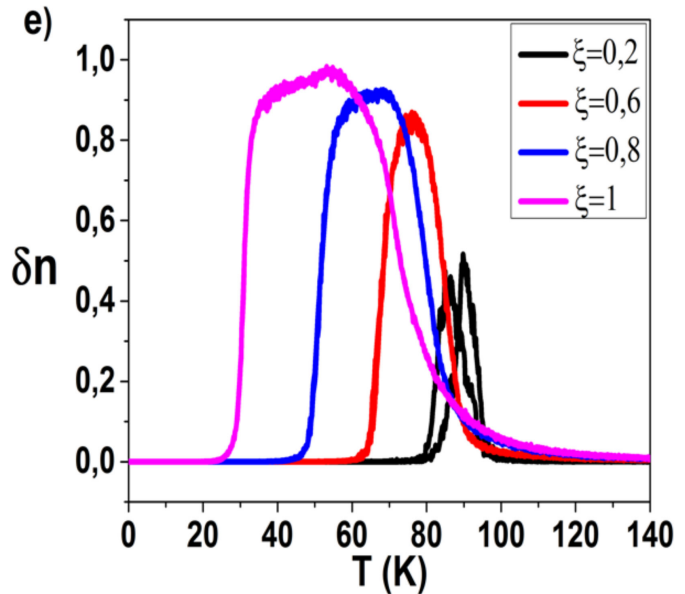

(b)

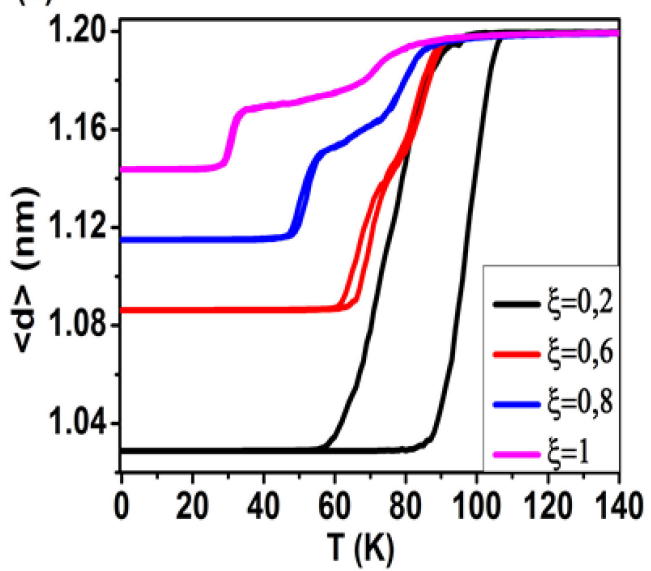

(d)

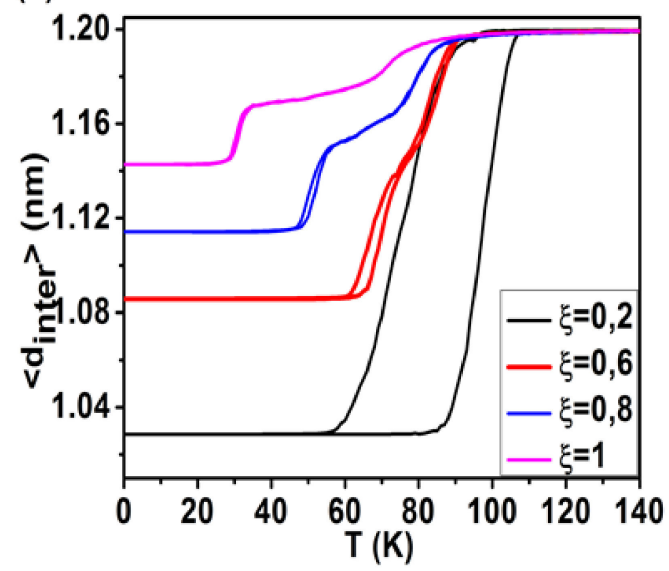

f)

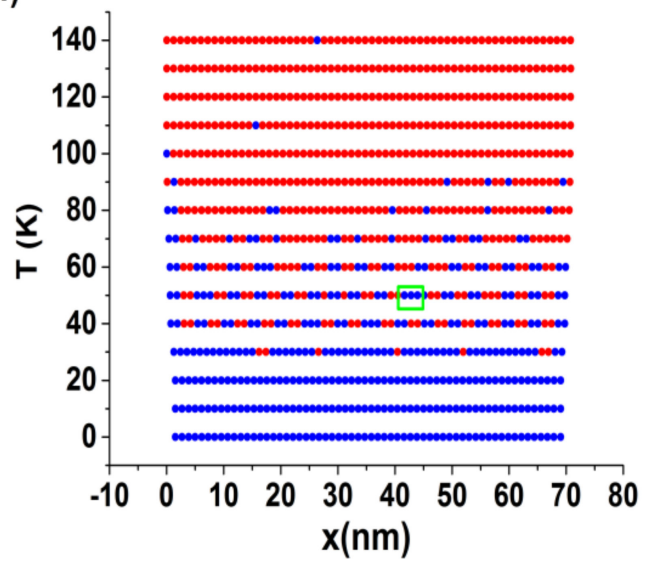

Figure 3. (a) Thermal dependence of the HS fraction obtained by MC simulations performed on spin and lattice positions of Hamiltonian (1) for different values of the elastic frustration strength $\xi$. First-order transition is obtained for weak $\xi$ (red and black curves), which transforms to two-step transitions with a plateau (pink and blue) for larger $\xi$. (b-d) Corresponding thermal dependence of the average $n n$ distances $\langle d\rangle$, and average $n n$ intra-binuclear, $\left\langle d_{\text {intra }}\right\rangle$, and inter-binuclear, $<d_{\text {inter }}>$, respectively. (e) Thermal-dependence of $\left.\delta n=\frac{1}{2}\left(<S_{i+1}^{\mathrm{A}}-S_{i}^{\mathrm{A}}\right\rangle\right)$, showing a symmetry breaking in the plateau region, where the spin states self-organize in the ordered state HS $=$ HS-LS $=$ LS ... for which $\delta n=1$. The degree of organization is partial for $\xi=0.6,0.8$ and becomes almost complete for $\xi=1$. (f) Spatial distribution of the spin states (red $=$ HS and blue $=$ LS) along the thermal transition for $\xi=1$, showing a perfect self-organization of the spin states in the plateau region, particularly at $40 \mathrm{~K}$. The parameter values are $R_{0}^{L L}=1 \mathrm{~nm}, R_{0}^{H H}=1.2 \mathrm{~nm}, R_{0}^{H L}=1.1 \mathrm{~nm}, \delta_{R}=0.2 \mathrm{~nm}$, $k_{1}^{\text {intra }}=k_{1}^{\text {inter }}=34,000 \mathrm{~K} \cdot \mathrm{nm}^{-2}, k_{2}^{\text {inter }}=\frac{k_{1}^{\text {intra }}}{10}, \Delta_{0}=450 \mathrm{~K}, \ln g=5$. 


\subsection{Analytical Expressions of the Relaxed Lattice Bond Lengths}

This section is devoted to the analytical investigations of the $\xi$ dependence of the relaxed $n n$ distance, $d_{x}$, for the three ordered electronic configurations of Figure 3, namely $\mathrm{H} 2 \mathrm{H} 2(\mathrm{HS}=\mathrm{HS}-\mathrm{HS}=\mathrm{HS} \ldots$ ), L2L2 (LS = LS-LS = LS $\ldots$ ) and $\mathrm{L} 2 \mathrm{H} 2(\mathrm{LS}=\mathrm{LS}-\mathrm{HS}=\mathrm{HS} \ldots$ ), corresponding respectively to a system fully HS, fully LS and antiferro-magnetical between binuclear units. When the system is fully HS, its elastic energy is always zero because the $n n$ and $n n n$ distances are not frustrated. In contrast, when the system adopts the LS and the ordered antiferro-like structures, the frustration appears, and their total relaxed elastic energies remain non-zero, due to the intrinsic incompatibility between their $n n$ and nnn distances.

For this, we consider a homogenous elastic system, in which we replace the local $n n$ (resp. nnn) distances $r_{i, i+1}$ (resp. $r_{i, i+2}$ ) by an invariant distance $x$ (resp. $y$ ) in the lattice [83]. We denote by $x_{L 2 L 2}^{\text {relax }}$ and $x_{L 2 H 2}^{\text {relax }}$ (resp. $y_{L 2 L 2}^{\text {relax }}$ and $y_{L 2 H 2}^{\text {relax }}$ ) the relaxed $n n$ (resp. $n n n$ ) distances corresponding to L2L2 and L2H2 macroscopic states. The total non-relaxed energies of the system (including electronic and elastic contributions) for the configurations H2H2, L2L2 and $\mathrm{L} 2 \mathrm{H} 2$ become,

$$
\begin{gathered}
E_{H 2 H 2}=N \Delta_{e f f}+\frac{(N-1)}{2} k_{1}^{\text {intra }}\left(x-R_{e q}^{n n}(+1,+1)\right)^{2}+\frac{(N-1)}{2} k_{1}^{\text {inter }}\left(y-R_{e q}^{n n}(+1,+1)\right)^{2} \\
+(N-2) k_{2}^{\text {inter }}\left(x+y-R_{e q}^{n n n}(+1,+1)\right)^{2} \\
E_{L 2 L 2}=-N \Delta_{e f f}+\frac{(N-1)}{2} k_{1}^{\text {intra }}\left(x-R_{e q}^{n n}(-1,-1)\right)^{2}+\frac{(N-1)}{2} k_{1}^{\text {inter }}\left(y-R_{e q}^{n n}(-1,-1)\right)^{2} \\
+(N-2) k_{2}^{\text {inter }}\left(x+y-R_{e q}^{\text {nnn }}(-1,-1)\right)^{2} \\
E_{L 2 H 2}=\frac{(N-1)}{4} k_{1}^{\text {intra }}\left(x-R_{e q}^{n n}(+1,+1)\right)^{2}+\frac{(N-1)}{2} k_{1}^{\text {inter }}\left(y-R_{e q}^{n n}(+1,-1)\right)^{2} \\
+\frac{(N-1)}{4} k_{1}^{\text {intra }}\left(x-R_{e q}^{n n}(-1,-1)\right)^{2}+(N-2) k_{2}^{\text {inter }}\left(x+y-R_{e q}^{n n n}(+1,-1)\right)^{2}
\end{gathered}
$$

For a chain with homogenous lattice interspacing $(x=y)$ and homogenous $n n$ elastic constant $k_{1}=k_{1}^{\text {intra }}=k_{1}^{\text {inter }}$, the total non-relaxed energy density after substituting $R_{e q}^{n n}\left(S_{i}, S_{j}\right)$ and $R_{e q}^{n n n}\left(S_{i}, S_{k}\right)$ by their expressions given in Equations (3)-(5), is given in the case of a long chain $(N \gg 1)$ by

$$
\frac{E_{L 2 L 2}}{N}=-\Delta_{e f f}+k_{1}^{\text {intra }}\left(x-R_{0}^{L L}-\delta_{R} \xi\right)^{2}+k_{2}^{\text {inter }}\left(2 x-2 R_{0}^{L L}\right)^{2}
$$

$$
\begin{gathered}
\frac{E_{L 2 H 2}}{N}=\frac{k_{1}^{\text {intra }}}{4}\left(x-R_{0}^{L L}-\delta_{R} \xi\right)^{2}+\frac{k_{1}^{\text {intra }}}{4}\left(x-R_{0}^{H H}\right)^{2}+\frac{k_{1}^{\text {intra }}}{2}\left(x-R_{0}^{H L}-\frac{\delta_{R} \xi}{2}\right)^{2}+k_{2}^{\text {inter }}\left(2 x-2 R_{0}^{H L}\right)^{2} \\
\frac{E_{H 2 H 2}}{N}=\Delta_{e f f}+\left(k_{1}^{\text {intra }}+4 k_{2}^{\text {inter }}\right)\left(x-R_{0}^{H H}\right)^{2}
\end{gathered}
$$

The relaxed distances are obtained by minimizing these expressions with respect to $x$, which gives,

$$
\begin{gathered}
x_{H 2 H 2}^{\text {relax }}=R_{0}^{H H}, \\
x_{L 2 L 2}^{\text {relax }}=R_{0}^{L L}+\frac{\delta_{R}}{1+4 k} \xi \\
x_{L 2 H 2}^{\text {relax }}=R_{0}^{H L}+\frac{\delta_{R}}{1+4 k} \frac{\xi}{2},
\end{gathered}
$$

where $k=\frac{k_{2}^{\text {inter }}}{k_{1}^{\text {intra }}}$ is the ratio between the $n n n$ and $n n$ elastic constants.

In Equations (11b) and (11c), we see that the relaxed $n n$ distances $\left(x_{L 2 L 2}^{r e l a x}\right)$ in the LS and in the ordered antiferro-like L2H2 state $\left(x_{\mathrm{L} 2 \mathrm{H} 2}^{\text {relax }}\right)$, are bigger than their respective nonfrustrated values, $R_{0}^{L L}$ and $R_{0}^{H L}$, and are simply linear increasing functions of the frustration 
parameter $\xi>0$ and the lattice parameter misfit $\delta_{R}$, while they are decreasing functions of the elastic constant ratio $k$.

Figure 4 displays the $\xi$-dependence of the analytical values of $x_{L L}^{\text {relax }}(\xi)$, given by Equation (11b) together with the results of MC simulations of Figure 3, at $0 \mathrm{~K}$. An excellent agreement is obtained between these two sets of data, confirming the relevance of the present analytical approach.

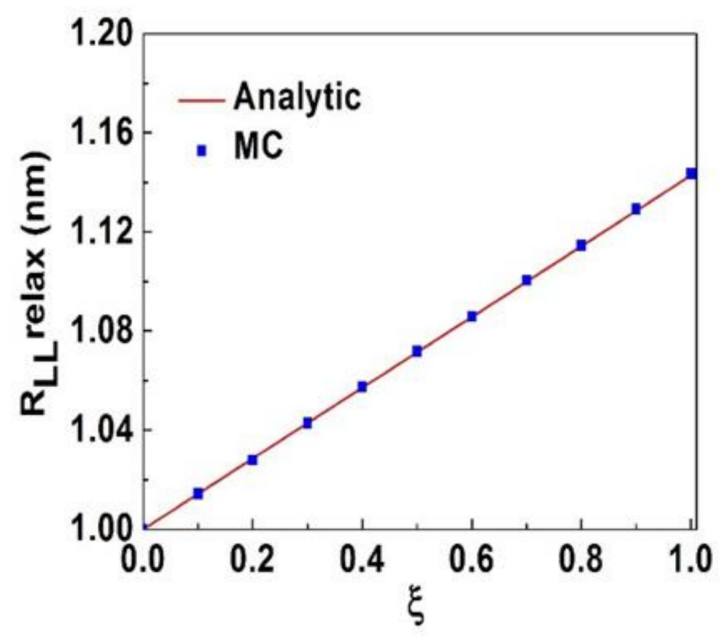

Figure 4. The $\xi$ dependance of the relaxed $n n$ distance, $R_{L L}^{\text {relax }}$, in the LS state. The blue squares are MC data and the full red line corresponds to the analytical prediction of Equation (11b). The calculations are performed at $T=10 \mathrm{~K}$. Observe the excellent quantitative predictions of the analytical approach.

\subsection{Effect of the Elastic Frustration on the Transition Temperatures and Phase Diagram}

In this section, we aim to find the analytical dependence of the transition temperature on the frustration parameter $\xi$. Two situations corresponding to the case of first-order transition and double step transitions, reported in Figure 3, are analyzed. In the latter case, we will express the $\xi$ dependence of the transition temperatures between the LS and the intermediate H2L2 state on the one hand, as well as that of the second transition temperature between the H2L2 state and the full HS state on the other hand.

We notice that in the case of the first-order transition with a thermal hysteresis, the analytical method will not give the limiting temperatures $\left(T^{-}, T^{+}\right)$of the hysteresis, which depend on the kinetic aspects of the process (lifetime of metastable states), but will rather lead to find the equilibrium temperature $T_{e q}$, while the $\mathrm{MC}$ simulations do exactly the contrary. In this last case, the equilibrium temperature is derived from MC data as $\sim\left(T^{-}+T^{+}\right) / 2$.

To determine analytically the transition temperatures, $T_{e q}$, as a function of the strength of frustration $\xi$, we study the case of the hysteretic transition of Figure 3a. This cooperative transformation between full LS and HS states takes place between two ordered states, namely LS and HS, in which their corresponding configurational entropies are null. Therefore, the condition of equal free energies at the transition temperature transforms to that of equal energies, and we then have $E\left(T=T_{\text {eq }},<S_{i}>=+1, x=x_{\text {relax }}^{H H}\right)=E\left(T=T_{\text {eq }},<S_{i}>=-1, x=x_{\text {relax }}^{L L}\right)$. Using the total energies calculted in Equation (7), we arrive after some simplifications to the following expression of the transition temperature,

$$
T_{e q}(\xi)=\frac{\Delta_{0}}{k_{\mathrm{B}} \ln g}-\frac{2}{k_{\mathrm{B}} \ln g} \frac{k_{2}^{\text {inter }}}{(1+4 k)} \delta_{R}^{2} \xi^{2}
$$

In Equation (12), the first term corresponds to the transition temperature of the usual Ising-like model and the second corresponds to the contribution of the elastic frustration which appears as an additional effective ligand field energy, $-\frac{2}{k_{\mathrm{B}} \ln g} \frac{k_{2}^{\text {inter }}}{(1+4 k)} \delta_{R}^{2} \xi^{2}$, to $\Delta_{0}$. It 
is also observed that the global transition temperature of the system shows a quadratic decrease with the frustration parameter.

The results are shown in the region (i) of the phase diagram of Figure 5, where we see that the analytical curve $T_{e q}(\xi)$ is in good agreement with the data of MC simulations.

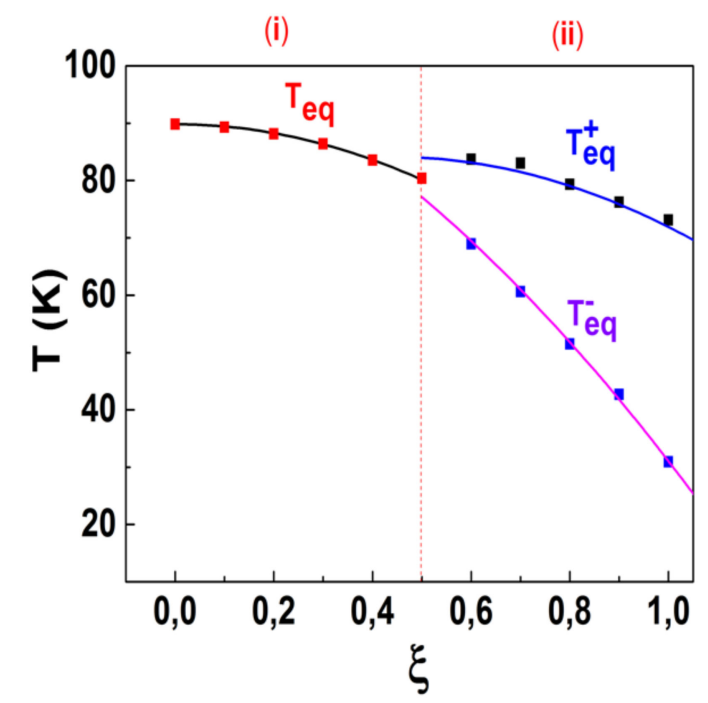

Figure 5. Phase diagram $T(\xi)$ summarizing the evolution of the equilibrium transition temperatures with the frustration parameter $\xi$. Squares represent the MC data and the continuous lines correspond to the analytical calculations. Region (i) with $0.2<\xi<0.5$ relates to the single-step first-order spin transition between HS and LS states, while region (ii) with $0.5<\xi<1$ delimits the complete two-step transitions behavior made of two consecutive gradual transitions. Black (resp. blue) and blue (resp. violet) squares (resp. lines) are MC (resp. analytical) data of $T_{e q}^{-}(\xi)$ and $T_{e q}^{+}(\xi)$.

\subsection{Condition of Appearance of the Two-Step Spin Transitions}

According to the results of Figure 3a, for large values of $\xi$, the thermal hysteresis transforms from first-order to a two-step transition with the emergence of a plateau around $n_{H S} \sim 0.5$. In this region, the spin states show an antiferromagnetic-like self-organization (L2H2L2 ... ) between the binuclear units.

As before, we calculate the analytical expressions of the transition temperatures, $T_{e q}^{-}(\xi)$ and $T_{e q}^{+}(\xi)$, corresponding to the transition between the full LS state and the intermediate $\mathrm{L} 2 \mathrm{H} 2$ state, and between the $\mathrm{L} 2 \mathrm{H} 2$ state and the full HS state. At these temperatures, the total energies of the elastically relaxed LL, L2H2 and HH states must obey the following conditions,

$$
\begin{gathered}
E\left(T=T_{\text {eq }}^{+},\left\langle S_{i}>=+1, x=x_{\text {relax }}^{H H}\right)=E\left(T=T_{\text {eq }}^{+},\left\langle S_{i}>=0, x=x_{\text {relax }}^{L 2 H 2}\right)\right.\right. \\
\quad \text { and } \\
E\left(T=T_{\text {eq }}^{-},\left\langle S_{i}>=-1, x=x_{\text {relax }}^{L L}\right)=E\left(T=T_{\text {eq }}^{-},\left\langle S_{i}>=0, x=x_{\text {relax }}^{L 2 H 2}\right)\right.\right.
\end{gathered}
$$

Using the expressions of energies found in Equations (10a)-(10c), we arrive immediately after some simplifications to,

$$
\begin{gathered}
T_{e q}^{+}(\xi)=T_{e q}(\xi)-\frac{2 \delta_{R}^{2}}{k_{\mathrm{B}} \ln g k_{1}^{\text {intra }}}\left[\frac{16 k^{2}+1}{16(1+4 k)^{2}}-\frac{k}{1+4 k}\right] \xi^{2}+\frac{k_{1}^{\text {intra }} \delta_{R}^{2}}{k_{\mathrm{B}} \ln g} \frac{\left(32 k^{2}+16 k+2\right)}{16(1+4 k)^{2}} \xi \\
-\frac{k_{1}^{\text {intra }} \delta_{R}^{2}}{16 k_{\mathrm{B}} \ln g} \frac{\left(22 k^{2}+16 k+2\right)}{(1+4 k)^{2}}
\end{gathered}
$$

and

$$
\begin{gathered}
T_{e q}^{-}(\xi)=T_{e q}(\xi)+\frac{2 \delta_{R}^{2}}{k_{\mathrm{B}} \ln g k_{1}^{\text {intra }}}\left[\frac{16 k^{2}+1}{16(1+4 k)^{2}}-\frac{k}{1+4 k}\right] \xi^{2}-\frac{k_{1}^{\text {intra }} \delta_{R}^{2}}{k_{\mathrm{B}} \ln g} \frac{\left(32 k^{2}+16 k+2\right)}{16(1+4 k)^{2}} \xi \\
+\frac{k_{1}^{\text {intra }} \delta_{R}^{2}}{16 k_{\mathrm{B}} \ln g} \frac{\left(22 k^{2}+16 k+2\right)}{(1+4 k)^{2}}
\end{gathered}
$$


The two-step transitions exist when the two following conditions are fulfilled: $T_{e q}^{+}(\xi)>T_{e q}^{-}(\xi)$ and $T_{e q}^{-}(\xi)>0$. In Figure 5, which represents the system's phase diagram of the system, we report both analytical $T_{e q}(\xi)$ curves and $\mathrm{MC}$ results. It is found that there is very good agreement between the analytical predictions and the MC data. In particular, two important regions of $\xi$ values $(\xi<0.5$ and $\xi>0.5)$ corresponding to first-step and two-step transitions are well reproduced as well as the "critical" value, $\xi \cong 0.5$, beyond which a "bifurcation-like" behavior of the transition temperature is evidenced.

\subsection{Effect of the Intramolecular Elastic Constant}

We now turn to the analysis of the effect of intramolecular elastic interactions inside the binuclear units on the global behavior of the chain, and for that we consider the last frustrated SCO chain with the parameter of frustration $\xi=1$. We fix the values of the $n n$ and $n n n$ intermolecular elastic constant to $k_{1}^{\text {inter }}=34,000 \mathrm{~K} \cdot \mathrm{nm}^{-2}$ and $k_{2}^{\text {inter }}=0.1 k_{1}^{\text {inter }}$ and we vary the intramolecular elastic constant, $k_{1}^{\text {intra }}$. We denote by $\eta=\frac{k_{1}^{\text {intra }}}{k_{1}^{\text {inter }}}$ the ratio between the intra and inter $n n$ elastic constants. The values of the parameters taken in the simulations are the same as the previous ones.

a/Thermal dependence of the HS fraction and lattice bond lengths:

In Figure 6a-e we show the behavior of $n_{H S}(T),<d>(T),<d_{\text {intra }}>(T),<d_{\text {inter }}>(T)$ and $\delta n$ curves for different values of the ratio between intra and intermolecular constants $\eta=\frac{k_{1}^{\text {intra }}}{k_{1}^{\text {inter }}}$. In the first figure, we see that increasing $\eta$ (i.e., $k_{1}^{\text {intra }}$ ) leads to an increase in the width of the plateau for the two-step transition, which reaches a saturation for the high values of $\eta$. It is also observed (see Figure 6) that at a low temperature, the $n n$ intramolecular distances increase and saturate rapidly to the value of the HS equilibrium distance while the intermolecular $n n$ distances decrease when $\eta$ increases. This is a result of the counterreaction of the increase in the intramolecular distance by maintaining the $n n n$ distance free from frustration.

Figure $6 \mathrm{f}$ presents the spatial configurations of the spin states (red dots $=\mathrm{HS}$ and blue dots $=$ LS) inside the lattice of the chain along the thermal transition for $\xi=1$ and $\eta=40$, giving rise to the two-step behavior. We see again the presence of the self-organized spin state structure L2H2 in the plateau region, i.e., for $26 \leq T \leq 45 \mathrm{~K}$. In addition, antiphase boundaries are also evidenced because the symmetric configuration (H2L2) has the same energy.

Now we are interested in the $\eta$ dependence of all $n n$ distances inside and between the binuclear units. For this, we select the three ordered electronic configurations of Figure 6, namely H2H2 (HS = HS-HS = HS ... ), L2L2 (LS = LS-LS = LS ...) and L2H2 (LS = LS-HS = HS ... ).

We denote by $x_{L L}^{\text {relax }}$ and $x_{L 2 H 2}^{\text {relax }}$ the relaxed $n n$ distances corresponding to L2L2 and L2H2 macroscopic states. We denote by $x$ the $n n$ intramolecular distances and by $y$ the $n n$ intermolecular distances. The total non-relaxed energy of the system is still given by Equations (9a)-(9c) for all these configurations.

Substituting $R_{e q}^{n n}\left(S_{i}, S_{j}\right)$ and $R_{e q}^{n n n}\left(S_{i}, S_{k}\right)$ by their expressions given in Equations (3)-(5), the elastic energy densities in the case of a long chain $(N \gg 1)$ are,

$$
\begin{gathered}
\frac{E_{H H}}{N}=\Delta_{e f f}+\frac{k_{1}^{\text {intra }}}{2}\left(x-R_{0}^{H H}\right)^{2}+\frac{k_{1}^{\text {inter }}}{2}\left(y-R_{0}^{H H}\right)^{2}+k_{2}^{\text {inter }}\left(x+y-2 R_{0}^{H H}\right)^{2} \\
\frac{E_{H H}}{N}=\Delta_{e f f}+\frac{k_{1}^{\text {intra }}}{2}\left(x-R_{0}^{H H}\right)^{2}+\frac{k_{1}^{\text {inter }}}{2}\left(y-R_{0}^{H H}\right)^{2}+k_{2}^{\text {inter }}\left(x+y-2 R_{0}^{H H}\right)^{2} \\
\frac{E_{L 2 H 2}}{N}=\frac{k_{1}^{\text {intra }}}{4}\left(x-R_{0}^{H H}\right)^{2}+\frac{k_{1}^{\text {inter }}}{2}\left(x-R_{0}^{H L}-\frac{\delta_{R} \xi}{2}\right)^{2}+\frac{k_{1}^{\text {intra }}}{4}\left(y-R_{0}^{L L}-\delta_{R} \xi\right)^{2}+k_{2}^{\text {inter }}\left(x+y-2 R_{0}^{H L}\right)^{2}
\end{gathered}
$$


(a)

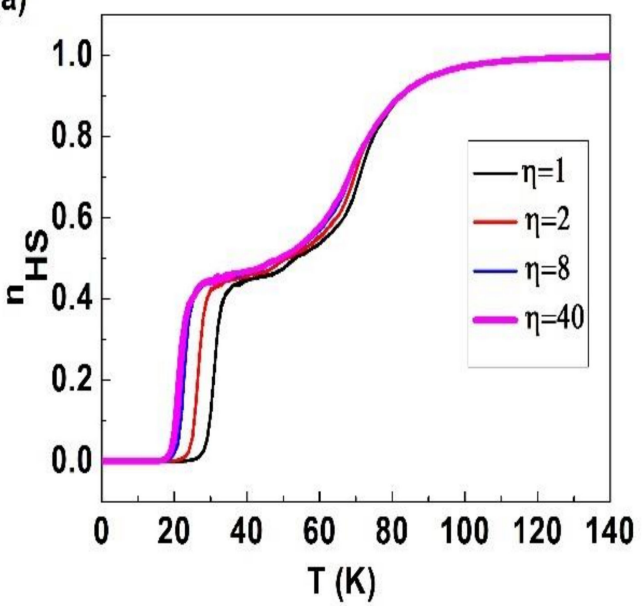

(c)

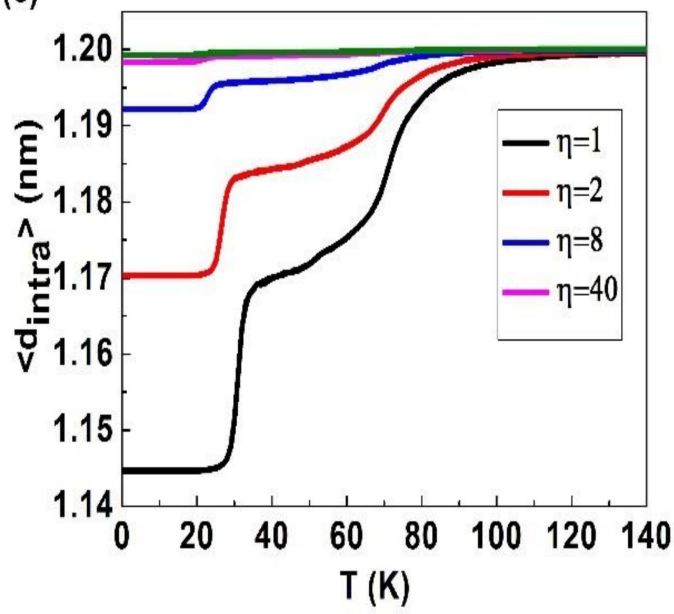

e)

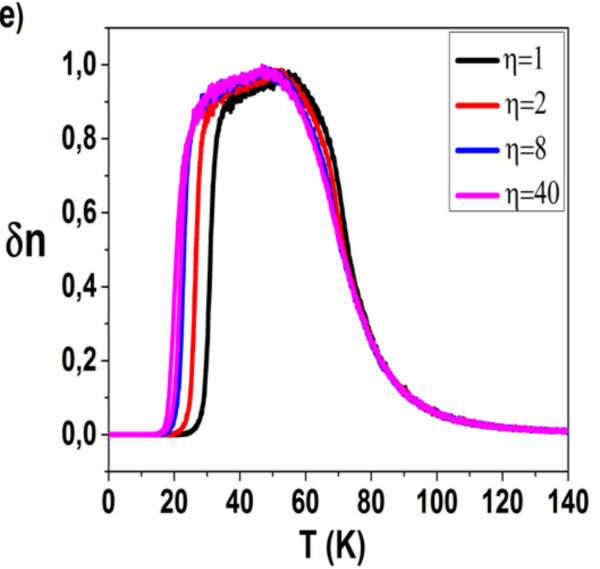

(b)

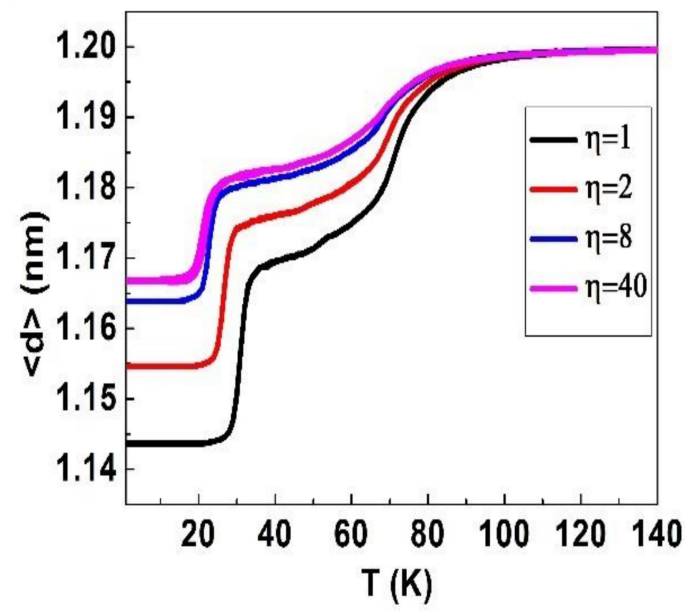

(d)

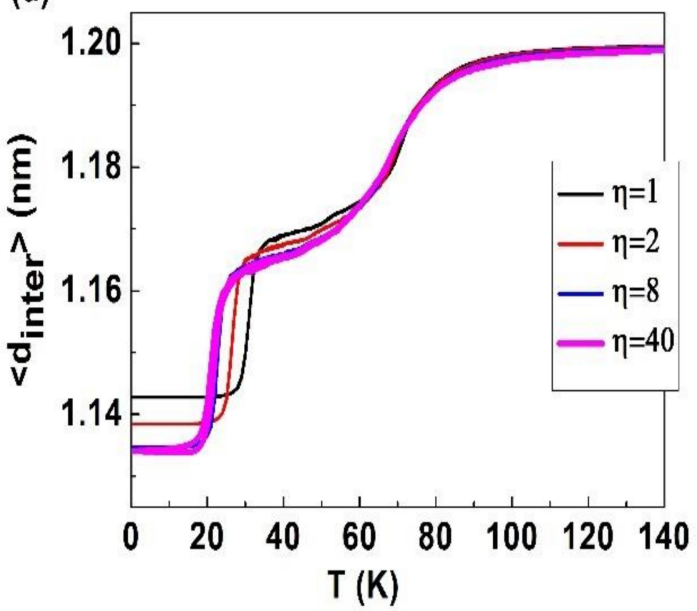

f)

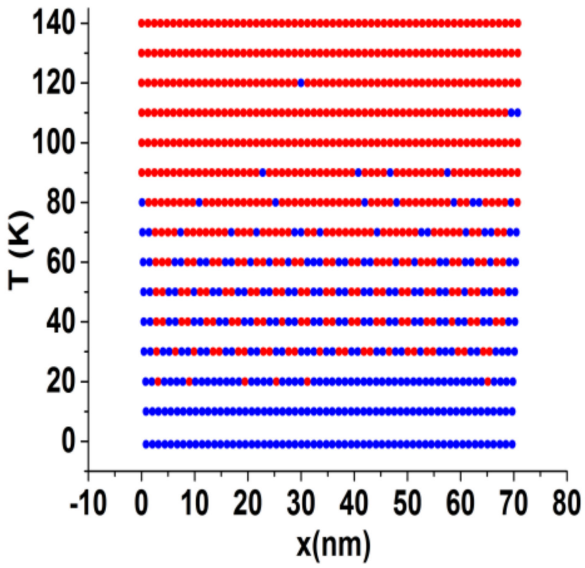

Figure 6. (a) Thermal dependence of the HS fraction obtained by MC simulations for $\eta$ values showing the occurrence of twostep transitions with a plateau at $\xi=1$, in which the width increases with increasing $\eta$ values. (b-d) corresponding thermal dependences of the average $n n$ distances over the lattice, $\langle d\rangle$, intra-binuclear, $\left\langle d_{\text {intra }}\right\rangle$ and inter-binuclear distances $\left\langle d_{\text {inter }}\right\rangle$, respectively. (e) Thermal dependence of $\delta n=\frac{1}{2}\left(\left\langle S_{i+1}^{\mathrm{A}}-S_{i}^{\mathrm{A}}\right\rangle\right)$ for different values of the elastic constant ratio $\eta$, showing a perfect order in the temperature interval, 40-60 K. (f) Snapshots of the lattice along the spin transition (red = HS and blue $=$ LS) showing a self-organization of the spin states of the binuclear units (HS = HS-LS = LS-HS = HS-LS ... ) in the plateau region $(\sim 50 \mathrm{~K})$. The other parameter values are: $R_{0}^{L L}=1 \mathrm{~nm}, R_{0}^{H H}=1.2 \mathrm{~nm}, R_{0}^{H L}=1.1 \mathrm{~nm}, \delta_{R}=0.2 \mathrm{~nm}$, $\xi=1, k_{1}^{\text {inter }}=34,000 \mathrm{~K} \cdot \mathrm{nm}^{-2}, k_{2}^{\text {inter }}=0.1 k_{1}^{\text {inter }}, \Delta_{0}=450 \mathrm{~K}$ and $\ln g=5$. 
The relaxed intra and inter distances are obtained by minimizing the three energy densities with respect to $x$ and $y$ parameters, i.e., $\frac{\partial(E / N)}{\partial x}=0$ and $\frac{\partial(E / N)}{\partial y}=0$. This leads to,

$$
\begin{aligned}
& \left\langle d_{\text {intra }}\right\rangle_{L L}=x_{\text {relax }}^{L L}=R_{0}^{L L}+\frac{\mathrm{A} \eta-\mathrm{B}}{\mathrm{A} \eta+\mathrm{B}} \delta_{R} \xi \\
& \left\langle d_{\text {inter }}\right\rangle_{L L}=y_{\text {relax }}^{L L}=R_{0}^{L L}+\frac{\mathrm{C} \eta+\mathrm{B}}{\mathrm{A} \eta+\mathrm{B}} \delta_{R} \xi
\end{aligned}
$$

and

$$
\begin{aligned}
& \left\langle d_{\text {intra }}\right\rangle_{L 2 H 2}=x_{\text {relax }}^{L 2 H 2}=R_{0}^{H L}+\frac{\mathrm{A} \eta-\mathrm{B}}{\mathrm{A} \eta+\mathrm{B}} \frac{\delta_{R} \xi}{2} \\
& \left\langle d_{\text {inter }}\right\rangle_{L 2 H 2}=y_{\text {relax }}^{L 2 H 2}=R_{0}^{H L}+\frac{\mathrm{C} \eta+\mathrm{B}}{\mathrm{A} \eta+\mathrm{B}} \frac{\delta_{R} \xi}{2}
\end{aligned}
$$

where $\mathrm{A}=1+2 k, \mathrm{~B}=2 k$ and $C=1-2 k$, with $k=\frac{k_{2}^{\text {inter }}}{k_{1}^{\text {inter }}}$.

The analytical $\eta$ dependence of $\left\langle d_{\text {intra }}\right\rangle,\left\langle d_{\text {inter }}\right\rangle$ and $\langle d\rangle=\frac{\left\langle d_{\text {intra }}\right\rangle+\left\langle d_{\text {inter }}\right\rangle}{2}$ for the three studied configurations are given in Figure 7 together with the MC results, showing a very good agreement between these two sets of data.

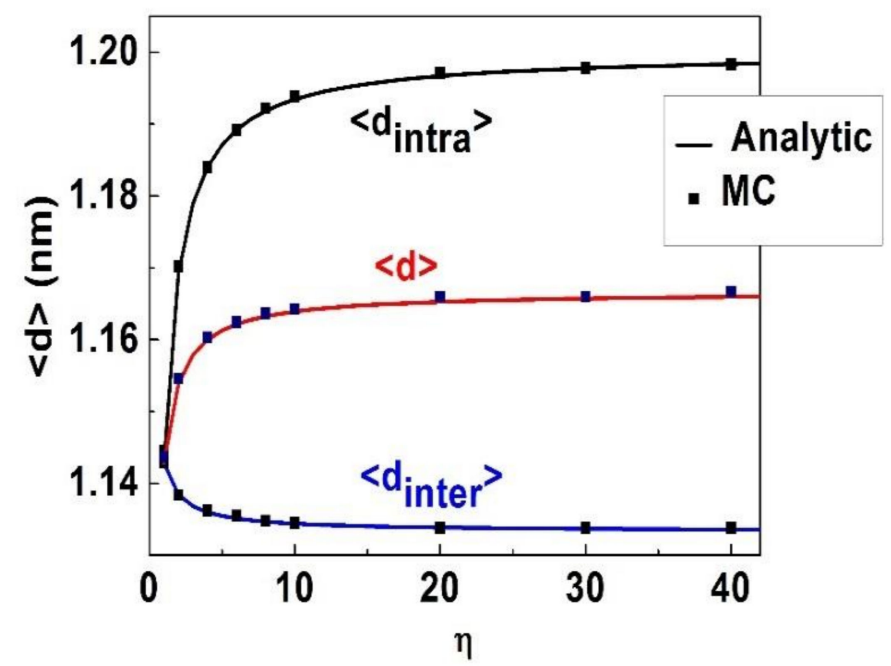

Figure 7. Relaxed $n n$ distance, $R_{L L}^{\text {relax }}$, in the LS state vs. elastic ratio $\eta$ between intra- and interbinuclear elastic constants. The red, blue and black squares are MC data and the black, red and blue lines correspond to the analytical prediction of Equations (16a)-(16d). The calculations are performed at $T=0 \mathrm{~K}$. Observe the excellent quantitative predictions of the analytical approach.

b/Transition temperatures and phase diagram:

The thermal dependence of the HS fraction of Figure 6a shows that for large values of $\eta$, the width of the plateau increases. In the following, we determine analytically the expressions of the transition temperatures, $T_{e q}^{-}(\eta)$ and $T_{e q}^{+}(\eta)$, associated with the $\mathrm{L} 2 \mathrm{~L} 2 \leftrightarrow \mathrm{L} 2 \mathrm{H} 2$ and the $\mathrm{H} 2 \mathrm{H} 2 \leftrightarrow \mathrm{L} 2 \mathrm{H} 2$ transitions, respectively. At these temperatures, the total energies of the elastically relaxed L2L2, L2H2 and $\mathrm{H} 2 \mathrm{H} 2$ states must respect the following conditions,

$$
\begin{aligned}
& \left.E\left(T=T_{e q}^{+},<S_{i}\right\rangle=+1, x_{\text {intra }}=\left\langle d_{\text {intra }}\right\rangle_{H H}, x_{\text {inter }}=\left\langle d_{\text {inter }}\right\rangle_{H H}\right) \\
= & E\left(T=T_{e q}^{+},<S_{i}>=0, x_{\text {intra }}=\left\langle d_{\text {intra }}\right\rangle_{L 2 H 2}, x_{\text {inter }}=\left\langle d_{\text {inter }}\right\rangle_{L 2 H 2}\right) \\
= & E\left(T=T_{e q}^{-},<S_{i}>=-1, x_{\text {intra }}=\left\langle d_{\text {intra }}\right\rangle_{L l}, x_{\text {inter }}=\left\langle d_{\text {inter }}\right\rangle_{L L}\right) \\
= & \left.E\left(T=T_{\text {eq }}^{-},<S_{i}\right\rangle=0, x_{\text {intra }}=\left\langle d_{\text {intra }}\right\rangle_{L 2 H 2}, x_{\text {inter }}=\left\langle d_{\text {inter }}\right\rangle_{L 2 H 2}\right)
\end{aligned}
$$


Using the expressions of energies found in Equations (15a)-(15c), we arrive immediately after some simplifications to,

$$
\begin{gathered}
T_{e q}^{-}(\eta)=\frac{\Delta_{0}}{k_{\mathrm{B}} \ln g}+\frac{k_{1}^{\text {inter }} \delta_{R}^{2}}{16(\mathrm{~A} \eta+\mathrm{B})^{2} k_{\mathrm{B}} \ln g}\left[\mathrm{~A}^{2}(\xi-1)^{2} \eta^{3}+\left(2 \mathrm{AB}\left(5 \xi^{2}-8 \xi+5\right)-16 \xi^{2}\left(\frac{(\mathrm{C}-\mathrm{A})^{2}}{2}+\frac{k^{\text {inter }}}{k_{1}^{\text {inter }}}(\mathrm{A}+\mathrm{C})^{2}\right)\right) \eta^{2}+\left(11 \xi^{2} \mathrm{~B}^{2}-6 \mathrm{~B}^{2}-31 \mathrm{~B}^{2}\right) \eta\right] \\
T_{e q}^{+}(\eta)=\frac{\Delta_{0}}{k_{\mathrm{B}} \ln g}-\frac{k_{1}^{\text {inter }} \delta_{R}^{2}}{16(\mathrm{~A} \eta+\mathrm{B})^{2} k_{\mathrm{B}} \ln g}\left[\mathrm{~A}^{2}(\xi-1)^{2} \eta^{3}+2 \mathrm{AB}\left(5 \xi^{2}-8 \xi+5\right) \eta^{2}+\left(11 \xi^{2} \mathrm{~B}^{2}-6 \xi^{2}+\mathrm{B}^{2}\right) \eta\right] .
\end{gathered}
$$

For $\xi=1$, which is the value considered in the MC investigation of $\eta$ effect, we find these temperatures,

$$
\begin{gathered}
T_{e q}^{-}(\eta)=\frac{\Delta_{0}}{k_{\mathrm{B}} \ln g}+\frac{k_{1}^{\text {inter }} \delta_{R}^{2}}{16(\mathrm{~A} \eta+\mathrm{B})^{2} k_{\mathrm{B}} \ln g}\left[\left(4 \mathrm{AB}-16\left(\frac{(\mathrm{C}-\mathrm{A})^{2}}{2}+\frac{k_{2}^{\text {inter }}}{k_{1}^{\text {inter }}}(\mathrm{A}+\mathrm{C})^{2}\right)\right) \eta^{2}-26 \mathrm{~B}^{2} \eta\right] \\
T_{e q}^{+}(\eta)=\frac{\Delta_{0}}{k_{\mathrm{B}} \ln g}-\frac{k_{1}^{\text {inter }} \delta_{R}^{2}}{16 k_{\mathrm{B}} \ln g(\mathrm{~A} \eta+\mathrm{B})^{2}}\left[4 \mathrm{AB} \eta^{2}+6 \mathrm{~B}^{2} \eta\right]
\end{gathered}
$$

Here again, the comparison between the analytical results and MC data, summarized in Figure 8, shows a very good agreement, confirming the relevance of the homogeneous elastic approach to reproduce the MC results.

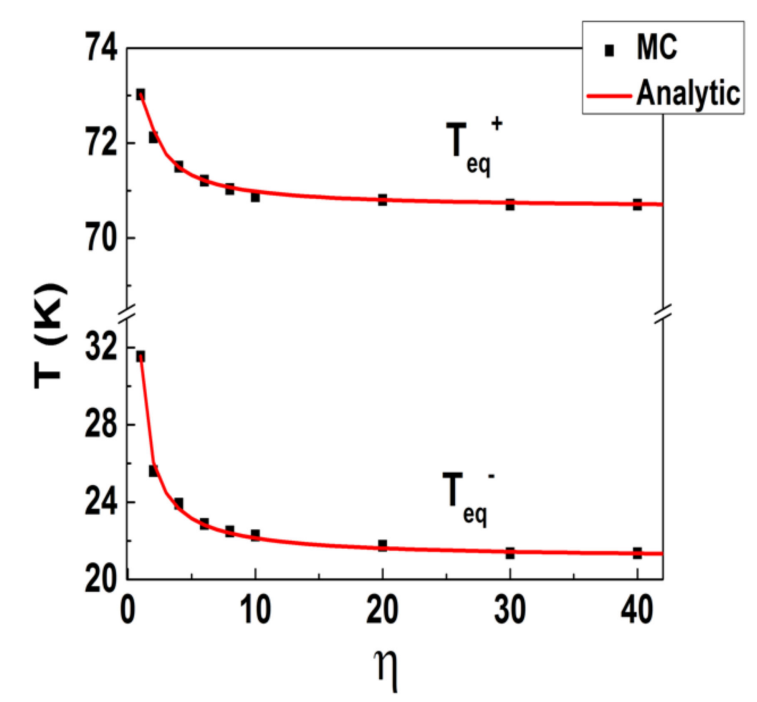

Figure 8. Elastic ratio $\eta$, dependence of the upper $\left(T_{e q}^{+}\right)$and lower $\left(T_{e q}^{-}\right)$transition temperatures of Figure 5 (a) obtained from MC (squares) simulations and analytical (continuous lines) investigations of Equations (19a) and (19b). An excellent agreement is found between both sets of data.

\section{Relaxation of the HS Fraction at Low Temperature}

This last section is devoted to the analysis of the dynamical properties of the present binuclear chain through the analysis of the time evolution of the HS fraction and the average $n n$ distances along the relaxation at low temperature $(10 \mathrm{~K})$ of the metastable HS state. For this, the chain is initially prepared in the HS state by setting all spin state values equal to +1 and all $n n$ distances equal to $R_{0}^{H H}$. In this state, by construction the chain does not experience any elastic frustration, which means that its elastic energy is equal to zero. Since at $10 \mathrm{~K}$, the LS state is the stable one (see Figure 3a), the HS chain will relax towards the LS state. Here, we are interested in the effect of the frustration parameter, $\xi$, and elastic interactions ratio, $\eta$, on the shape of the relaxation curves. 


\subsection{Elastic Frustration Effects on the Low Temperature Relaxation of the Metastable HS State}

We start by analyzing the effect of the elastic frustration on a homogeneous binuclear chain in which all involved (intra- and intermolecular) elastic constants are equal. Here, the values of the $n n$ elastic constants have been fixed to $k_{1}^{\text {intra }}=k_{1}^{\text {inter }}=51,000 \mathrm{~K} \cdot \mathrm{nm}^{-2}$, and those of $n n n$ are taken as $k_{2}^{\text {inter }}=0.1 k_{1}^{\text {inter }}$. The obtained results are summarized in Figure $9 a, b$, where we observe that for the values of frustration parameter, $0<\xi<0.6$, the lifetime of the HS metastable state decreases and the relaxation process accelerates with increasing $\xi$ values. This behavior is ascribed to the decrease in the effective elastic energy barrier facing the flip of an HS site to LS. Indeed, for $\xi=0$, the elastic energy cost for the HS to LS spin flip is easily found as $\Delta E_{0}=\left(\frac{1}{2} k_{1}^{\text {intra }}+\frac{1}{2} k_{1}^{\text {inter }}+k_{2}^{\text {inter }}\right) \delta_{R}^{2}$, where $\delta_{R}=\left(R_{0}^{H H}-R_{0}^{L L}\right)$. When $\xi$ increases, the average $n n$ distance in the LS state is no more $R_{0}^{L L}$, but becomes $x_{L L}^{\text {relax }}=R_{0}^{L L}+\frac{\delta_{R}}{1+4 k} \xi$ (see Equation (12b)), which is bigger than $R_{0}^{L L}$, as we can observe in Figure $9 b$, where the value of $\langle d\rangle$ at infinite time raises up as $\xi$ increases. Consequently, the new energy barrier becomes $\Delta E(\xi)=\left(\frac{1}{2} k_{1}^{\text {intra }}+\frac{1}{2} k_{1}^{\text {inter }}+k_{2}^{\text {inter }}\right)\left(R_{0}^{H H}-x_{L L}^{\text {relax }}\right)^{2}=\left(1-\frac{1}{1+4 k} \xi\right)^{2} \Delta E_{0}$. This energy barrier has a non-monotonous behavior with $\xi$, since it goes through a minimum for $\xi=1+4 k$. It has a decreasing function in the interval $0<\xi<1+4 k$ and its derivative becomes positive for $\xi>1+4 k$. In the situation in Figure 9, the ratio, $k=\frac{k_{2}^{\text {inter }}}{k_{1}^{\text {intra }}}=\frac{1}{10}$, and the barrier, $\Delta E$, is then expected to completely vanish for $\xi=0.7 . \Delta E(\xi)$, is then clearly lower than $\Delta E_{0}$ in the region $\xi<0.7$ and has a decreasing behavior as $\xi$ increases. As a result, the relaxation of the metastable HS state is expected to be faster for higher $\xi$ values in this region. This analytical prediction is in excellent agreement with the MC data in Figure 9a,b.

On the other hand, for $\xi>0.74$, Figure $9 \mathrm{c}, \mathrm{d}$ exhibit two relaxation regimes. In the first regime, both $n_{H S}$ and $\left\langle d>\right.$ fall down to intermediate values $\left(n_{H S}=0.4\right.$ and $\langle d\rangle \sim 1.14$ ), and then a new regime in which the relaxation process slows down starts from these values. In Figure 9e we plot the temporal evolution of the average intradinuclear spin-spin correlation, $\left\langle S_{\mathrm{A}} S_{\mathrm{B}}\right\rangle$, for different values of the elastic frustration strength $\xi(0.75<\xi<0.83)$, which shows the same trend as those of $n_{H S}$ and $<d>$. Since, $<S_{\mathrm{A}} S_{\mathrm{B}}>=n_{++}+n_{--}-2 n_{+-}$where $n_{++}, n_{--}$and $n_{+-}=n_{-+}$are the respective probabilities of obtaining HS-HS, LS-LS and HS-LS dimers; it is clear that the first regime of $<S_{\mathrm{A}} S_{\mathrm{B}}>$ corresponds to the formation of HS-LS dimers. Thus, for $\xi=0.82$ and 0.83 , for example, $<S_{\mathrm{A}} S_{\mathrm{B}}>$ reaches the value 0.2 , which means that $80 \%\left(=n_{+-}+n_{-+}\right)$of the lattice is formed by HS-LS dimers and $20 \%$ of LS-LS dimers. This leads to $n_{H S}=0.41$ at the end of the first regime, in excellent agreement with the results of Figure 9c. This means that in the first regime, for strong $\xi$ values, the chain tends to stabilize transient antiferro-elastic intra-binuclear states. In the second regime, $\left\langle S_{\mathrm{A}} S_{\mathrm{B}}>\right.$ increases due to the increase in the LS-LS species as a result of the conversion of HS-LS binuclear configurations to LS-LS states, due to thermal relaxation. These behaviors are confirmed by the time dependence of the snapshots of Figure 9f, which clearly indicate the emergence of new and transient organized states identified around 800 MCS and 1000 MCS, and resemble a mixture of successive $(\mathrm{H}=\mathrm{L})-(\mathrm{H}=\mathrm{L})$ or $(\mathrm{L}=\mathrm{H}$ and $(\mathrm{H}=\mathrm{L})-(\mathrm{L}=\mathrm{L})$... configurations.

In this second slow regime, taking place after $1000 \mathrm{MCS}$, the increase in $\xi$ results in an increase in the lifetime of the metastable state. This behavior is in very good agreement with the previous analysis based on the non-monotonous behavior of the elastic energy barrier with the frustration parameter, $\xi$. 

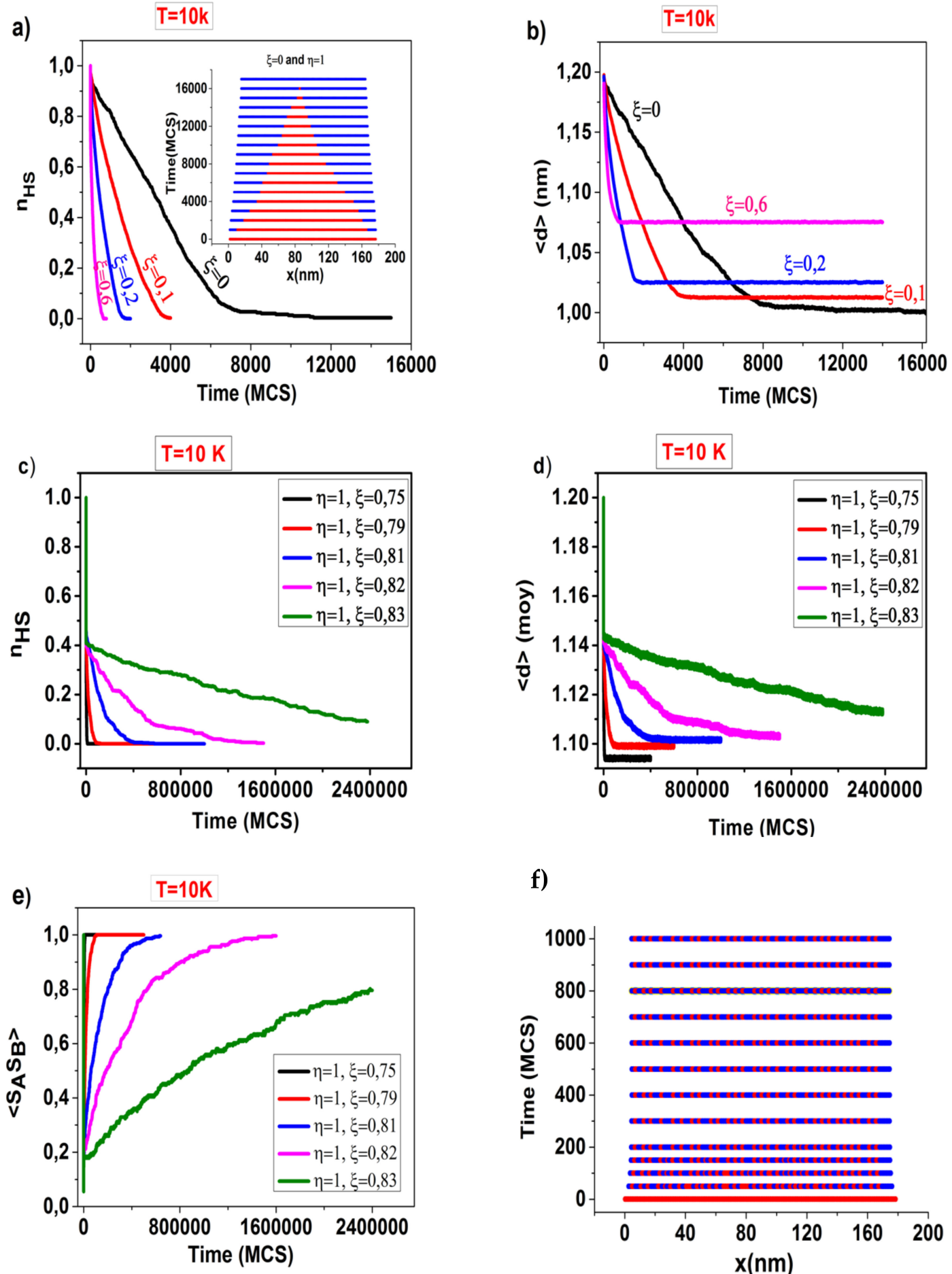

f)

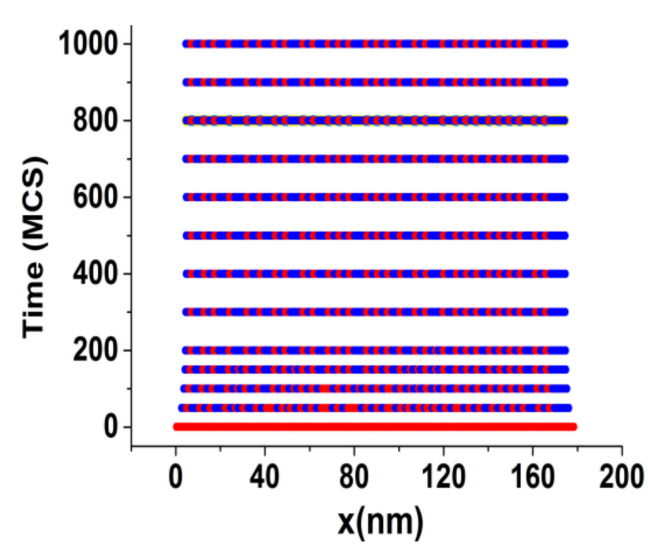

Figure 9. (a) MC results of the time evolution of the isothermal relaxation of the metastable HS fraction, $n_{H S}$, for different values of the elastic frustration parameter $\xi$ (for $0<\xi<0.6$ ) with $\eta=1$. Inset: corresponding snapshots of the lattice along the relaxation (red $=$ HS and blue $=$ LS) of the homogenous binuclear chain for $\xi=0$, which corresponds to the black curve in Figure 9a. (b) Corresponding time evolution of the average $n n$ distances $\langle d\rangle$. (c) Time evolution of the HS fraction, $n_{H S}$ (for $0.75<\xi<0.83$ ). (d) Corresponding time evolution of the average $n n$ distances $<d>$. The parameter values are $R_{0}^{L L}=1 \mathrm{~nm}, R_{0}^{H H}=1.2 \mathrm{~nm}, R_{0}^{H L}=1.1 \mathrm{~nm}, \delta_{R}=0.2 \mathrm{~nm}, k_{1}^{\text {intra }}=k_{1}^{\text {inter }}=51,000 \mathrm{~K} \cdot \mathrm{nm}^{-2}, k_{2}^{\text {inter }}=\frac{k_{1}^{\text {intra }}}{10}$, $\Delta_{0}=450 \mathrm{~K}$ and $\ln g=5$. (e) Temporal evolution of the average intramolecular spin-spin correlation, $\left\langle S_{\mathrm{A}} S_{\mathrm{B}}\right\rangle$, for different values of the elastic frustration strength $\xi(0.75<\xi<0.83)$ showing the existence of two relaxation regimes. (f) Spin configurations along the relaxation of the chain for $\xi=0.82$ and $\eta=1$ (red = HS and blue $=\mathrm{LS}$ ), showing the emergence of a transient self-organized state between 800 MCS and 1000 MCS, made of a mixture of successive $(H=L)-(H=L)$ and $(\mathrm{L}=\mathrm{L})$ configurations. 


\subsection{Effect of Intramolecular Elastic Constant on the Relaxation Curves}

Here we study the effect of intramolecular elastic interactions inside the binuclear units on the relaxation process of the previous chain. For this, we monitor the ratio, $\eta=\frac{k_{1}^{\text {intra }}}{k_{1}^{\text {inter }}}$, between the intra and intermolecular elastic constants in the range 1-1.7. The values of the other parameters are the same as those in Figure 9.

Figure 10a,b displays the time dependence of the HS fraction, $n_{H S}$, as well as that of the average $n n$ bond length distance, $\langle d\rangle$, along the relaxation process from HS to LS at $\mathrm{T}=10 \mathrm{~K}$ for different values of $\eta$. Both figures show that an increasing $\eta$ leads to an increase in the lifetime of the HS metastable, which extend the relaxation process with an emergence of a slight plateau for $\eta=1.7$. The corresponding snapshots for the case $\eta=1.7$ are given in Figure 11.
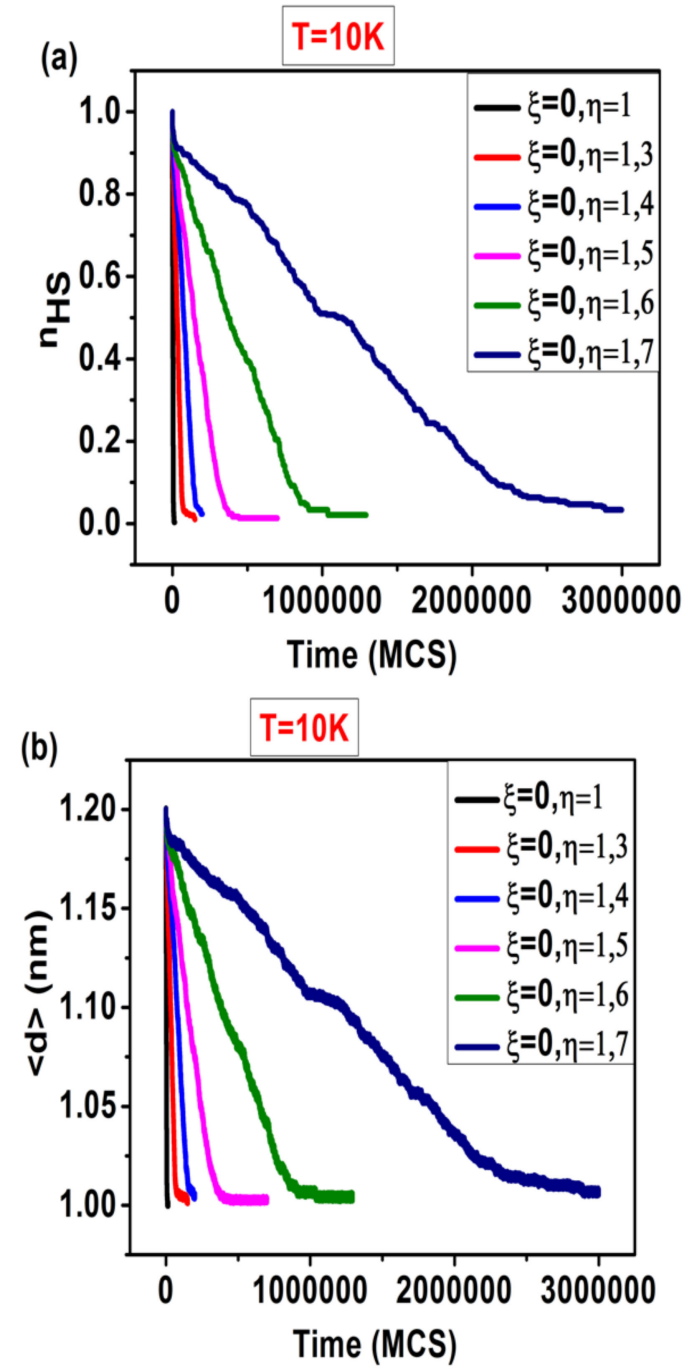

Figure 10. (a) MC results of the time evolution of the HS fraction $n_{H S}$ of Hamiltonian (1) for different values of the elastic ratio, $\eta$, along the relaxation of the metastable HS fraction of non-frustrated binuclear SCO chain containing 60 binuclear units, showing the occurrence of two-step transitions with a plateau at $\eta=1.7\left(n_{H S}=0.53\right)$ (dark blue curve). (b) Corresponding thermal dependences of the average $n n$ distance, $\langle d\rangle$. The other parameter values are the same as those in Figure 9. 


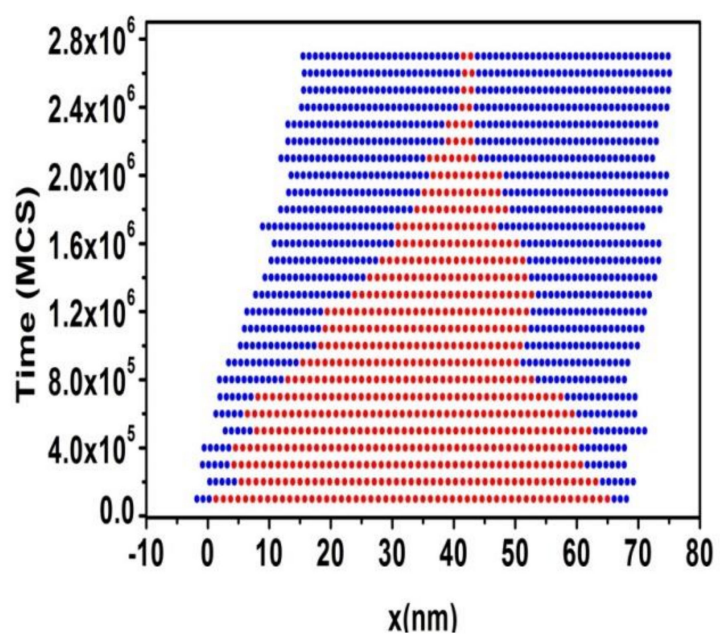

Figure 11. Snapshots of the lattice along the relaxation (red $=$ HS and blue $=$ LS) for $\xi=0$, $\eta=1.7$ and $\mathrm{T}=10 \mathrm{~K}$. The other parameter values are: $R_{0}^{L L}=1 \mathrm{~nm} R_{0}^{L L}=1 \mathrm{~nm}, R_{0}^{H H}=1.2 \mathrm{~nm}$, $R_{0}^{H L}=1.1 \mathrm{~nm}, \delta_{R}=0.2 \mathrm{~nm}, \xi=1, k_{1}^{\text {inter }}=34,000 \mathrm{~K} \cdot \mathrm{nm}^{-2}, k_{2}^{\text {inter }}=0.1 k_{1}^{\text {inter }}, \Delta_{0}=450 \mathrm{~K}$ and $\ln g=5$.

\section{Conclusions}

$1 \mathrm{D}$ binuclear SCO chains are widely explored in the literature due to a rich variety of behaviors, among them being first-order, gradual, two-step as well as incomplete or partial spin transitions. However, the elastic description of 1D binuclear SCO chains has never been investigated in the theoretical literature of SCO materials. To address this shortcoming, we have extended the previous electroelastic model and adapted it to mimic the thermal behavior of a 1D elastic chain made of elastically-coupled binuclear spin-crossover blocks, $\cdots \mathrm{A}=\mathrm{B}-\mathrm{A}=\mathrm{B}-\mathrm{A}=\mathrm{B} \cdots$. To account for the steric effects inside and between the binuclear units, we included an elastic frustration leading to antagonist equilibrium distances between nearest and next nearest neighbors. The sites of the binuclear unit interact via a harmonic intramolecular spring and the binuclear unit itself couples to the neighboring binuclear units via other springs. We have solved this model by combining MC simulations performed on the spin states and another MC procedure for the lattice positions. To understand the relevant parameters acting on the outputs of the model, we have also adapted an analytical method, developed in the context of recent studies of frustrated 1D chains [62]. By doing so, we could predict the dependence of the HS fraction on several model parameters, such as the elastic frustration strength and the intramolecular elastic constant.

In the present work, we have first demonstrated that the injection of an elastic frustration between $n n$ and $n n n$ equilibrium distances, i.e., inside the binuclear units, as a parameter leading to expand the intramolecular distance in the LS state, leads to stabilize intermediate spin states along the thermal transition between the LS and the HS phases. We found that according to the strength of this elastic frustration, well organized antiferroelastic structures (LS = LS-HS $=$ HS-LS $=$ LS . . ) ) are stabilized. This result is contrasting with the usual antiferro HS-LS-HS-LS . . configurations which appeared in the previous frustrated 1D mononuclear model [62]. However, if one considers the binuclear unit as a single component, then it becomes clear that the binuclear unit organizes itself in the plateau under the usual HS-LS-HS-LS antiferro configurations. We find that the spin state organization in the plateau is made of self-organized structures. In the second step of this work, we identified that the intramolecular elastic interaction linking the atoms inside the binuclear units plays a leading role in the thermal properties of the studied spin transition between the LS and the HS phases. Thus, an increase in this elastic constant with respect to the intermolecular elastic constant results in two-step transition behaviors with an increase in the width of the plateau which reaches saturation. On the other hand, the stabilization 
of the previous LS = LS-HS = HS-LS = LS ... configuration expresses that in this situation the two sites inside the binuclear are so strongly correlated that each binuclear unit reacts as a single molecular object.

We have also studied the time dependence of the HS fraction and lattice parameter of the present frustrated chain, thus simulating the relaxation of a metastable photoinduced HS state. The obtained results clearly demonstrate that the shape of the relaxation curves transform from continuous to two-step behaviors, which is reminiscent of the thermal dependence of the order parameters of the system at equilibrium thermodynamics.

Lastly, the present work opens several opportunities of extensions related first with the examination of other situations of frustrations. Indeed, here we considered the HS state free from frustration, but one may consider the LS state free from frustration and the HS and HS-LS states as frustrated. This will surely lead to other types of thermal behavior of the binuclear chain. Moreover, this model deserves to be extended to two-dimensions to well analyze the dimensionality effects on the modes of self-organization of the spin states.

Author Contributions: Conceptualization, K.B.; methodology, K.B.; software, K.B., R.T. and H.O.; writing-original draft preparation, K.B. and R.T.; editing, K.B., R.T. and H.O.; funding acquisition, K.B. All authors have read and agreed to the published version of the manuscript.

Funding: This research was funded by ANR (Agence Nationale de la Recherche Scientifique), grant number Mol-CoSM N ${ }^{\circ}$ ANR-20-CE07-0028-02 and the Universities of Versailles and Paris-SaclayUPSAY, the CNRS (Centre National de la Recherche Scientifique) and LIA (International Associate French Japan Laboratory). We thank all of them for their financial support.

Institutional Review Board Statement: Not applicable.

Informed Consent Statement: Not applicable.

Data Availability Statement: The data of this manuscript are available within the manuscript.

Conflicts of Interest: The authors declare no conflict of interest.

\section{References}

1. Phan, H.; Benjamin, S.M.; Steven, E.; Brooks, J.S.; Shatruk, M. Photomagnetic Response in Highly Conductive Iron(II) Spin Crossover Complexes with TCNQ Radicals. Angew. Chem. Int. Ed. 2015, 54, 823. [CrossRef]

2. Ohkoshi, S.I.; Tokoro, H. Photomagnetism in Cyano-Bridged Bimetal Assemblies. Acc. Chem. Res. 2012, 45, 1749. [CrossRef]

3. Coronado, E.; Galán-Mascarós, J.R.; Monrabal-Capilla, M.; García-Martínez, J.; Pardo-Ibáñez, P. Bistable Spin-Crossover Nanoparticles Showing Magnetic Thermal Hysteresis near Room Temperature. Adv. Mater. 2007, 19, 1359. [CrossRef]

4. Kahn, O.; Jay-Martinez, C. Spin-Transition Polymers: From Molecular Materials Toward Memory Devices. Science 1998, $279,44$. [CrossRef]

5. Gütlich, P.; Hauser, A.; Spiering, H. Thermal and Optical Switching of Iron(II) Complexes. Angew. Chem. Int. Ed. 1994, 33, 2024-2054. [CrossRef]

6. Gütlich, P.; Goodwin, H.A. Spin Crossover in Transition Metal Compounds, I, II, III; Gütlich, P., Goodwin, H.A., Eds.; Springer: Berlin/Heidelberg, Germany, 2004; p. 233.

7. Halcrow, M.A. Spin-Crossover Materials-Properties and Applications; John Wiley \& Sons: Chichester, UK, 2013; Volume 52, p. 10419. [CrossRef]

8. Garcia, Y.; Kahn, O.; Ader, J.-P.; Buzdin, A.; Meurdesoif, Y.; Guillot, M. The effect of a magnetic field on the inversion temperature of a spin crossover compound revisited. Phys. Lett. A. 2000, 271, 145-154. [CrossRef]

9. Bousseksou, A.; Boukheddaden, K.; Goiran, M.; Consejo, C.; Boillot, M.-L.; Tuchagues, J.-P. Dynamic response of the spin-crossover solid $\mathrm{Co}\left(\mathrm{H}_{2}(\mathrm{fsa})_{2} \mathrm{en}\right)(\mathrm{py})_{2}$ to a pulsed magnetic field. Phys. Rev. B 2002, 65, 172412. [CrossRef]

10. Pillet, S.; Hubsch, J.; Lecomte, C. Single crystal diffraction analysis of the thermal spin conversion in $\left[\mathrm{Fe}(\mathrm{btr})_{2}\left(\mathrm{NCS}_{2}\right]\left(\mathrm{H}_{2} \mathrm{O}\right)\right.$ : Evidence for spinlike domain formation. Eur. Phys. J. B 2004, 38, 541-552. [CrossRef]

11. Tanasa, R.; Enachescu, C.; Stancu, A.; Linares, J.; Codjovi, E.; Varret, F.; Haasnoot, J. First-order reversal curve analysis of spin-transition thermal hysteresis in terms of physical-parameter distributions and their correlations. Phys. Rev. B 2005, 71, 014431. [CrossRef]

12. Olguín, J.; Brooker, S. Spin crossover active iron(II) complexes of selected pyrazole-pyridine/pyrazine ligands. Coord. Chem. Rev. 2011, 255, 203-240. [CrossRef]

13. Halcrow, M.A. Structure:function relationships in molecular spin-crossover complexes. Chem. Soc. Rev. 2011, 40, 4119-4142. [CrossRef] 
14. Bertoni, R.; Lorenc, M.; Cailleau, H.; Tissot, A.; Laisney, J.; Boillot, M.-L.; Stoleriu, L.; Stancu, A.; Enachescu, C.; Collet, E. Elastically driven cooperative response of a molecular material impacted by a laser pulse. Nat. Mater. 2016, 15, 606-610. [CrossRef]

15. Gütlich, P.; Gaspar, A.B.; Garcia, Y. Spin state switching in iron coordination compounds. Beilstein J. Org. Chem. 2013, 9, 342-391. [CrossRef]

16. Ridier, K.; Craig, G.A.; Damay, F.; Fennell, T.; Murrie, M.; Chaboussant, G. Probing photoinduced spin states in spin-crossover molecules with neutron scattering. Phys. Rev. B. 2017, 95, 094403. [CrossRef]

17. Brooker, S. Spin crossover with thermal hysteresis: Practicalities and lessons learnt. Chem. Soc. Rev. 2015, 44, 2880-2892. [CrossRef]

18. Jureschi, C.M.; Linares, J.; Rotaru, A.; Ritti, M.H.; Parlier, M.; Dîrtu, M.M.; Wolff, M.; Garcia, Y. Pressure Sensor via Optical Detection Based on a 1D Spin Transition Coordination Polymer. Sensors 2015, 15, 2388-2398. [CrossRef]

19. Decurtins, S.; Gütlich, P.; Hasselbach, K.M.; Hauser, A.; Spiering, H. Light-induced excited-spin-state trapping in iron(II) spin-crossover systems. Optical spectroscopic and magnetic susceptibility study. Inorgan. Chem. 1985, 24, 2174-2178. [CrossRef]

20. Hauser, A. Reversibility of light-induced excited spin state trapping in the Fe(ptz)6(BF4)2, and the Zn1-xFex(ptz)6(BF4)2 spin-crossover systems. Chem. Phys. Lett. 1986, 124, 543-548. [CrossRef]

21. Hauser, A.; Adler, J.; Gütlich, P. Light-induced excited spin state trapping (LIESST) in [Fe(2-mephen)3]2+ embedded in polymer matrices. Chem. Phys. Lett. 1988, 152, 468-472. [CrossRef]

22. Gütlich, P.; Garcia, Y.; Goodwin, H.A. Spin crossover phenomena in Fe(ii) complexesDedicated to Professor, F.A. Cotton on occasion of his 70th birthday. Chem. Soc. Rev. 2000, 29, 419-427. [CrossRef]

23. Hauser, A. Light-Induced Spin Crossover and the High-Spin $\rightarrow$ Low-Spin Relaxation. In Spin Crossover in Transition Metal Compounds; Chem, T.C., Ed.; Springer: New York, NY, USA, 2004; Volume 234, pp. 155-198.

24. Mahfoud, T.; Molnar, G.; Bonhommeau, S.; Cobo, S.; Salmon, L.; Demont, P.; Tokoro, H.; Ohkoshi, S.; Boukheddaden, K.; Bousseksou, A. Electric-Field-Induced Charge-Transfer Phase Transition: A Promising Approach Toward Electrically Switchable Devices. J. Am. Chem. Soc. 2009, 131, 15049-15054. [CrossRef]

25. Rotaru, A.; Dugay, J.; Tan, R.P.; Guralskiy, I.A.; Salmon, L.; Demont, P.; Carrey, J.; Molnar, G.; Respaud, M.; Bousseksou, A. Nanoelectromanipulation of Spin Crossover Nanorods: Towards Switchable Nanoelectronic Devices. Adv. Mater. 2013, 25, 1745-1749. [CrossRef]

26. Prins, F.; Monrabal-Capilla, M.; Osorio, E.A.; Coronado, E.; van der Zant, H.S. Room-Temperature Electrical Addressing of a Bistable Spin-Crossover Molecular System. Adv. Mater. 2011, 23, 1545-1549. [CrossRef]

27. Moussa, N.O.; Ostrovskii, D.; Garcia, V.M.; Molnár, G.; Tanaka, K.; Gaspar, A.B.; Real, J.A.; Bousseksou, A. Bidirectional photoswitching of the spin state of iron(II) ions in a triazol based spin crossover complex within the thermal hysteresis loop. Chem. Phys. Lett. 2009, 477, 156-159. [CrossRef]

28. Gallois, B.; Real, J.A.; Hauw, C.; Zarembowitch, J. Structural changes associated with the spin transition in bis(isothiocyanato)bis(1,10phenanthroline)iron: A single-crystal x-ray investigation. Inorg. Chem. 1990, 29, 1152. [CrossRef]

29. Shepherd, H.J.; Rosa, P.; Fallis, I.A.; Guionneau, P.; Howard, J.A.K.; Goeta, A.E. Structural origin of the gradual spin transition in a mononuclear iron(II) complex. Phys. Chem. Solids 2012, 73, 193-197. [CrossRef]

30. Takahashi, K.; Mori, H.; Kobayashi, H.; Sato, O. Mechanism of reversible spin transition with a thermal hysteresis loop in [FeIII(qsal)2][Ni(dmise)2] 2CH3CN: Selenium analogue of the precursor of an Fe(III) spin-crossover molecular conducting system. Polyhedron 2009, 28, 1776. [CrossRef]

31. Fedaoui, D.; Bouhadja, Y.; Kaiba, K.; Guionneau, P.; Létard, J. Complexation of 2,6-Bis(3-pyrazolyl)pyridine-Bis(thiocyanato)iron(II) with a Bridging 4,4'-Bipyridine: A New Example of a Dinuclear Spin Crossover Complex. Eur. J. Inorg. Chem. 2008, 7, $1022-1023$. [CrossRef]

32. De Gaetano, Y.; Jeanneau, E.; Verat, A.Y.; Rechignat, L.; Bousseksou, A.; Matouzenko, G.S. Ligand-Induced Distortions and Magneto-Structural Correlations in a Family of Dinuclear Spin Crossover Compounds with Bipyridyl-Like Bridging Ligands. Eur. J. Inorg. Chem. 2013, 2013, 1015-1023. [CrossRef]

33. Sciortino, N.F.; Scherl-Gruenwald, K.R.; Chastanet, G.; Halder, G.J.; Chapman, K.W.; Létard, J.F.; Kepert, C.J. Hysteretic Three-Step Spin Crossover in a Thermo-and Photochromic 3D Pillared Hofmann-type Metal-Organic Framework. Angew. Chem. Int. Ed. Engl. 2012, 124, 10301-10305. [CrossRef]

34. Romstedt, H.; Spiering, H.; Gütlich, P. Modelling of two step high spin $\rightleftharpoons$ low spin transitions using the cluster variation method. J. Phys. Chem. Solids 1998, 59, 1353-1362. [CrossRef]

35. Li, Z.Y.; Dai, J.W.; Shiota, Y.O.; Yoshizawa, K.; Kanegawa, S.; Sato, O. Multi-Step Spin Crossover Accompanied by Symmetry Breaking in an FeIII Complex: Crystallographic Evidence and DFT Studies. Chemistry. 2013, 19, 12948-12952. [CrossRef]

36. Vela, S.; Gourlaouen, C.; Fumanal, J.; Ribas-Arino, J. Disclosing the Ligand-and Solvent-Induced Changes on the Spin Transition and Optical Properties of Fe(II)-Indazolylpyridine Complexes. Magnetochemistry 2016, 2, 6. [CrossRef]

37. Floquet, S.; Salunke, S.; Boillot, M.L.; Clément, R.; Varret, F.; Boukheddaden, K.; Rivière, E. The Spin Transition of an Iron(III) Complex Intercalated in a MnPS3 Layered Magnet. Occurrence of a Hysteresis Effect on Removal of Lattice Solvent. Chem. Mater. 2002, 14, 4164-4171. [CrossRef]

38. Koike, M.; Murakami, K.; Fujinami, T.; Nishi, K.; Matsumoto, N.; Sunatsuki, Y. Syntheses, three types of hydrogen-bonded assembly structures, and magnetic properties of [FeIII(Him)2(hapen)]Y solvent $\left(\mathrm{Him}=\right.$ imidazole, hapen $=\mathrm{N}, \mathrm{N}^{\prime}$-bis $(2-$ 
hydroxyacetophenylidene)ethylenediamine, $\mathrm{Y}=\mathrm{BPh} 4-, \mathrm{CF} 3 \mathrm{SO} 3-, \mathrm{PF} 6-, \mathrm{ClO} 4-$, and BF4-). Inorg. Chim. Acta 2013, 399, 185-192. [CrossRef]

39. Helena, J.; Shepherdab, P.; Rosab, L.; Vendiera, N.; Casatic, J.F.; Letardb, A.; Bousseksou, P.; Guionneau, G.M. High-pressure spin-crossover in a dinuclear Fe(ii) complex. Phys. Chem. 2012, 14, 5265-5271. [CrossRef]

40. Real, J.A.; Bolvin, H.; Bousseksou, A.; Dworkin, A.; Kahn, O.; Varret, F.; Zarembowitch, J. Two-step spin crossover in the new dinuclear compound $\left[\mathrm{Fe}(\mathrm{bt})(\mathrm{NCS})_{2}\right]_{2}$ bpym, with bt $=2,2^{\prime}$-bi-2-thiazoline and bpym $=2,2^{\prime}$-bipyrimidine: Experimental investigation and theoretical approach. J. Am. Chem. Soc. 1992, 114, 4650-4658. [CrossRef]

41. Real, J.A.; Gaspar, A.B.; Muñoz, M.C.; Gütlich, P.; Ksenofontov, V.; Spiering, H. Bipyrimidine-Bridged Dinuclear Iron(II) Spin Crossover Compounds. Top. Curr. Chem. 2004, 233, 167.

42. Köppen, H.; Müller, E.; Köhler, C.; Spiering, H.; Meissner, E.; Gütlich, P. Unusual spin-transition anomaly in the crossover system [Fe(2-pic)3] Cl2_EtOH. Chem. Phys. Lett. 1982, 91, 348-352. [CrossRef]

43. Jakobi, R.; Spiering, H.; Gütlich, P. Thermodynamics of the spin transition in $\left[\mathrm{Fe}_{\mathrm{x}} \mathrm{Zn}_{1-\mathrm{x}}(2-\mathrm{pic})_{3}\right] \mathrm{Cl}_{2} \_$EtOH. J. Phys. Chem. Sol. 1988, 53, 267-275. [CrossRef]

44. Klein, Y.M.; Sciortino, N.F.; Ragon, F.; Housecroft, C.E.; Kepert, C.J.; Neville, S.M. Spin crossover intermediate plateau stabilization in a flexible 2-D Hofmann-type coordination polymer. Chem. Commun. 2014, 50, 3838. [CrossRef]

45. Ohtani, R.; Arai, M.; Hori, A.; Takata, M.; Kitao, S.; Seto, M.; Kitagawa, S.; Ohba, M. Modulation of Spin-Crossover Behavior in an Elongated and Flexible Hofmann-Type Porous Coordination Polymer. J. Inorg. Organomet. Polym. 2013, 23, 104-110. [CrossRef]

46. Cointe, M.B.; Moussa, N.O.; Trzop, E.; Moréac, A.; Molnar, G.; Toupet, L.; Bousseksou, A.; Létard, J.F.; Matouzenko, G.S. Symmetry breaking and light-induced spin-state trapping in a mononuclear FeII complex with the two-step thermal conversion. Phys. Rev. B. 2010, 82, 214106. [CrossRef]

47. Paez-Espejo, M.; Sy, M.; Boukheddaden, K. Elastic Frustration Causing Two-Step and Multistep Transitions in Spin-Crossover Solids: Emergence of Complex Antiferroelastic Structures. J. Am. Chem. Soc. 2016, 138, 3202-3210. [CrossRef]

48. Watanabe, H.; Tanaka, K.; Bréfuel, N.; Cailleau, H.; Létard, J.F.; Ravy, S.; Fertey, P.; Nishino, M.; Miyashita, S.; Collet, E. Ordering phenomena of high-spin/low-spin states in stepwise spin-crossover materials described by the ANNNI model. Phys. Rev. $B$ Condens. Mater. Phys. 2016, 93, 014419. [CrossRef]

49. Ortega-Villar, N.; Muñoz, M.; Real, J. Symmetry Breaking in Iron(II) Spin-Crossover Molecular Crystals. Magnetochemistry 2016, 2, 16. [CrossRef]

50. Trzop, E.; Zhang, D.; Piñeiro-Lopez, L.; Valverde-Muñoz, F.J.; Muñoz, M.C.; Palatinus, L.; Guerin, L.; Cailleau, H.; Real, J.A.; Collet, E. Inside Back Cover: First Step Towards a Devil's Staircase in Spin-Crossover Materials Angew. Chem. Int. Ed. 2016, 55, 8675. [CrossRef]

51. Murnaghan, K.D.; Carbonera, C.; Toupet, L.; Griffin, M.; Dirtu, M.M.; Desplanches, C.; Garcia, Y.; Collet, E.; Létard, J.F.; Morgan, G.G. Spin-State Ordering on One Sub-lattice of a Mononuclear Iron(III) Spin Crossover Complex Exhibiting LIESST and TIESST. Chem. Eur. J. 2014, 20, 5613. [CrossRef] [PubMed]

52. Li, Z.-Y.; Ohtsu, H.; Kojima, T.; Dai, J.-W.; Yoshida, T.; Breedlove, B.K.; Zhang, W.-X.; Iguchi, H.; Sato, O.; Kawano, M.; et al. Direct Observation of Ordered High-Spin-Low-Spin Intermediate States of an Iron(III) Three-Step Spin-Crossover Complex. Angew. Chem. Int. Ed. 2016, 55, 5184-5189. [CrossRef] [PubMed]

53. Hayami, S.; Nakaya, M.; Ohmagari, H.; Alao, A.S.; Nakamura, M.; Ohtani, R.; Yamaguchi, R.; Kuroda-Sowa, T.; Clegg, J.K Spin-crossover behaviors in solvated cobalt(ii) compounds. Dalton Trans. 2015, 44, 9345-9348. [CrossRef]

54. Bauer, W.; Pfaffeneder, T.; Achterhold, K.; Weber, B. Complete Two-Step Spin-Transition in a 1D Chain Iron (II) Complex with a 110-K Wide Intermediate Plateau. Eur. J. Inorg. Chem. 2011, 21, 3183-3192. [CrossRef]

55. Dirtu, M.M.; Schmit, F.; Naik, A.D.; Rusu, I.; Rotaru, A.; Rackwitz, S.; Wolny, J.A.; Schunemann, V.; Spinu, L.; Garcia, Y. Two-Step Spin Transition in a 1D FeII 1,2,4-Triazole Chain Compound Chem. Eur. J. 2015, 21, 5843-5855. [CrossRef]

56. Neville, S.M.; Leita, B.A.; Halder, G.J.; Kepert, C.J.; Moubaraki, B.; Letard, J.F.; Murray, K.S. Understanding the Two-Step Spin-Transition Phenomenon in Iron(II) 1D Chain Materials Chem. Eur. J. 2008, 14, 10123-10133. [CrossRef]

57. Sorai, M.; Seki, S. Phonon coupled cooperative low-spin 1A1high-spin 5T2 transition in [Fe(phen)2(NCS)2] and [Fe(phen)2(NCSe)2] crystals. J. Phys. Chem. Solids 1974, 35, 555-570. [CrossRef]

58. Wajnflasz, J.; Pick, R. Transition low spin»-« High spin in Fe2+ complex. J. Phys. Colloq. 1971, 32, C1-91-C1-92. [CrossRef]

59. Bousseksou, A.; Nasser, J.; Linares, J.; Boukheddaden, K.; Varret, F. Ising-like model for the two-step spin-crossover. J. Phys. I Fr. 1992, 2, 1381-1403. [CrossRef]

60. Nishino, M.; Miyashita, S.; Boukheddaden, K. Effective interaction range in the spin crossover phenomenon: Wajnflasz and domain models. J. Chem. Phys. 2003, 118, 4594. [CrossRef]

61. Boukheddaden, K.; Linares, J.; Codjovi, E.; Varret, F.; Niel, V.; Real, J.A. Dynamical ising-like model for the two-step spin-crossover systems. J. Appl. Phys. 2003, 93, 7103-7105. [CrossRef]

62. Traiche, R.; Sy, M.; Boukheddaden, K. Elastic Frustration in 1D Spin-Crossover Chains: Evidence of Multi-Step Transitions and Self-Organizations of the Spin States. J. Phys. Chem. C. 2018, 122, 4083-4096. [CrossRef]

63. Pillet, S.; Bendeif, E.E.; Bonnet, S.; Shepherd, H.J.; Guionneau, P. Multimetastability, phototrapping, and thermal trapping of a metastable commensurate superstructure in a Fe spin-crossover compound. Phys. Rev. B Condens. Matter Phys. 2012, 86, 064106. [CrossRef] 
64. Collet, E.; Watanabe, H.; Brefuel, N.; Palatinus, L.; Roudaut, L.; Toupet, L.; Tanaka, K.; Tuchagues, J.P.; Fertey, P.; Ravy, S.A. Aperiodic Spin State Ordering of Bistable Molecules and Its Photoinduced Erasing. Phys. Rev. Lett. 2012, 109, 257206. [CrossRef]

65. Gaspar, A.B.; Ksenofontov, V.; Reiman, S.; Gutlich, P.; Thompson, A.L.; Goeta, A.E.; Munoz, M.C.; Real, J.A. Mössbauer Investigation of the Photoexcited Spin States and Crystal Structure Analysis of the Spin-Crossover Dinuclear Complex [\{Fe(bt)(NCS)2\}2bpym] (bt = 2,2'-Bithiazoline, bpym = 2,2'-Bipyrimidine). Chem. Eur. J. 2006, 12, 9289-9298. [CrossRef]

66. Fitzpatrick, A.J.; Trzop, E.; Muller-Bunz, H.; Dirtu, M.M.; Garcia, Y.; Collet, E.; Morgan, G.G. Electronic vs. structural ordering in a manganese(iii) spin crossover complex. Chem. Commun. 2015, 51, 17540-17543. [CrossRef]

67. Chernyshov, D.; Hostettler, M.; Tornroos, K.W.; Burgi, H.B. Ordering Phenomena and Phase Transitions in a Spin-Crossover Compound-Uncovering the Nature of the Intermediate Phase of [Fe(2-pic)3]Cl2·EtOH. Angew. Chem. Int. Ed. 2003, 42, 3825-3830. [CrossRef]

68. Popa, A.; Stoleriua, L.; Enachescu, C. Tutorial on the elastic theory of spin crossover materials. J. Appl. Phys. 2021, $129,131101$. [CrossRef]

69. Azzolina, G.; Bertoni, R.; Collet, E. General Landau theory of non-symmetry-breaking and symmetry-breaking spin transition materials. J. App. Phys. 2021, 129, 085106. [CrossRef]

70. Slimani, A.; Boukheddaden, K.; Varret, F.; Oubouchou, H.; Nishino, M.; Miyashita, S. Microscopic spin-distortion model for switchable molecular solids: Spatiotemporal study of the deformation field and local stress at the thermal spin transition. Phys. Rev. 2013, 87, 014111. [CrossRef]

71. Boukheddaden, K.; Traiche, R.; Oubouchou, H.; Linares, J. Multistep Relaxations in a Spin-Crossover Lattice with Defect: A Spatiotemporal Study of the Domain Propagation. Magnetochemistry 2016, 2, 17. [CrossRef]

72. Enachescu, C.; Stoleriu, L.; Stancu, A.; Hauser, A. Model for Elastic Relaxation Phenomena in Finite 2D Hexagonal Molecular Lattices. Phys. Rev. Lett. 2009, 102, 257204. [CrossRef]

73. Enachescu, C.; Nishino, M.; Miyashita, S.; Stoleriu, L.; Stancu, A. Monte Carlo Metropolis study of cluster evolution in spincrossover solids within the framework of a mechanoelastic model. Phys. Rev. B 2012, 86, 054114. [CrossRef]

74. Nishino, M.; Miyashita, S. Effect of the short-range interaction on critical phenomena in elastic interaction systems. Phys. Rev. B 2013, 88, 014108. [CrossRef]

75. Enachescu, C.; Stoleriu, L.; Nishino, M.; Miyashita, S.; Stancu, A.; Lorenc, M.; Bertoni, R.; Cailleau, H.; Collet, E. Theoretical approach for elastically driven cooperative switching of spin-crossover compounds impacted by an ultrashort laser pulse. Phys. Rev. B. 2017, 95, 224107. [CrossRef]

76. Stoleriu, L.; Nishino, M.; Miyashita, S.; Stancu, A.; Hauser, A.; Enachescu, C. Cluster evolution in molecular three-dimensional spin-crossover systems. Phys. Rev. B 2017, 96, 064115. [CrossRef]

77. Nishino, M.; Miyashita, S.; Rikvold, P.A. Nontrivial phase diagram for an elastic interaction model of spin crossover materials with antiferromagnetic-like short-range interactions. Phys. Rev. B 2017, 96, 144425. [CrossRef]

78. Enachescu, C.; Nicolazzi, W. Elastic models, lattice dynamics and finite size effects in molecular spin crossover systems. Comptes Rendus Chim. 2018, 21, 1179-1195. [CrossRef]

79. Sorai, M. Heat Capacity Studies of Spin Crossover Systems; Springer: Berlin/Heidelberg, Germany, 2004; Volume 235, pp. 135-170.

80. Singh, Y.; Oubouchou, H.; Nishino, M.; Miyashita, S.; Boukheddaden, K. Elastic-frustration-driven unusual magnetoelastic properties in a switchable core-shell spin-crossover nanostructure. Phys. Rev. B 2020, 101, 054105. [CrossRef]

81. Miyashita, S.; Konishi, Y.; Nishino, M.; Tokoro, H.; Rikvold, P.A. Realization of the mean-field universality class in spin-crossover materials. Phys. Rev. B Condens. Matter Mater. Phys. 2008, 77, 014105. [CrossRef]

82. Boukheddaden, K.; Nishino, M.; Miyashita, S.; Varret, F. Molecular Dynamics and Transfer Integral Investigations of an Elastic Anharmonic Model for Phonon-Induced Spin Crossover. Phys. Rev. Lett. 2008, 100, 177206. [CrossRef]

83. Ndiaye, M.; Belmouri, N.; Linares, J.; Boukheddaden, K. Elastic Origin of the Unsymmetrical Thermal Hysteresis in Spin Crossover Materials: Evidence of Symmetry Breaking. Symmetry 2021, 13, 828. [CrossRef] 